\title{
One-step approach to ARPES from strongly correlated solids: a Mott-Hubbard system.
}

\author{
R.O. Kuzian ${ }^{1,2}$ and E.E. Krasovskii ${ }^{3,2,4}$ \\ ${ }^{1}$ Institute for Problems of Materials Science NASU, Krzhizhanovskogo 3, 03180 Kiev, Ukraine \\ ${ }^{2}$ Donostia International Physics Center (DIPC), Paseo Manuel de Lardizabal 4, \\ San Sebastián/Donostia, 20018 Basque Country, Spain \\ ${ }^{3}$ Departamento de Física de Materiales, Facultad de Ciencias Químicas, \\ Universidad del Pais Vasco/Euskal Herriko Unibertsitatea, \\ Apdo. 1072, San Sebastián/Donostia, 20080 Basque Country, Spain \\ ${ }^{4}$ IKERBASQUE, Basque Foundation for Science, 48013 Bilbao, Spain
}

(Dated: May 27, 2022)

\begin{abstract}
An expression is derived for angle-resolved photocurrent from a semi-infinite correlated system. Within the sudden approximation, the photocurrent is proportional to the spectral function of a one-particle two-time retarded Green's function $\mathcal{G}$ of an operator that creates an electron in a special quantum state $\chi$ localized at the surface. For a system described by a many-body single-band model we present an analytical expression that relates the Green's function $\mathcal{G}$ with the Green's function of an infinite crystal $G_{b, \mathbf{k}}(\omega)$ in Wannier representation. The role of final states and of the crystal surface is analysed for a model Green's function of the infinite crystal with a three-peak spectral function typical of a Mott-Hubbard metal. The momentum dependence of both the quasiparticle pole position and the spectral weight of the incoherent band manifest themselves in the shape of the photocurrent energy distribution curve.
\end{abstract}

PACS numbers: 79.60.-i, 71.27.+a, 71.10.Fd, 73.20.-r

\section{INTRODUCTION}

Angle-resolved photoemission spectroscopy (ARPES) has proved an indispensable tool to study the electronic structure of solids ${ }^{1}$ [ 4 . It has become especially important with the discovery of high-Tc cuprate superconductors, when the enhanced experimental and theoretical effort was put into the studies of strongly correlated electron systems (SCES) ${ }^{516}$. Mean-field-based approaches fail to describe the valence band of SCES, so ARPES becomes a crucial source of information about the electronic structure and a verification tool for manybody theories ${ }^{7}$. However, the interpretation of ARPES in terms of one-electron many-body spectral function (SF) may be sufficient only when the energy dispersion perpendicular to the surface is of the order of or smaller than the experimental energy resolution. This is the case in the layered cuprates ${ }^{214}$ or perovskite-type vanadates ${ }^{811}$, which have a quasi-two-dimensional valence and conduction bands despite their cubic lattice. Still, most of correlated compounds have a three-dimensional electronic structure ${ }^{6}$, and the photohole dispersion normal to the surface requires a more thorough theoretical analysis of the ARPES.

A conclusive interpretation of the ARPES experiment depends on the knowledge of final states of the photoemission process. In the sudden approximation ${ }^{12}$, the final states are time-reversed low-energy electron diffraction (LEED) states ${ }^{13}$, which decay into the interior of the solid in accord with the surface sensitivity of ARPES. To be realistic, a proper calculation of the photocurrent should allow for changes of the electronic structure near the surface and include the excitation probabilities.
The most elaborate theoretical framework to deal with ARPES is the one-step theory 13. It describes the excitation, the transport of the photoelectron to the crystal surface, and the escape into the vacuum as a single quantum-mechanical process including all multiplescattering events. This approach allows to perform realistic photocurrent calculations based on Kohn-Sham eigenfunctions, and it is implemented in several computer codes $17 / 19120$.

The one-step approach was also formulated for nonlocal potentials 2122$]$, and in Refs. 23 and 24 it was combined with the dynamic mean field theory (DMFT) 25 within the Korringa-Kohn-Rostoker multiple scattering formalism. It was recently applied for the interpretation of photoemission spectra of $3 d$ metals 28 30. Most of studies of SCES are, however, performed within the basis of localized Wannier functions 31,33 , as originally proposed by P.W. Anderson ${ }^{34}$ and J. Hubbard ${ }^{355}$. The Wannier representation is quite natural here, since the largest Coulomb interaction term - the so-called Hubbard interaction, which is responsible for electron correlations in $d$ or $f$ shells of transition metals - is diagonal in this basis 35 . The theoretical many-body bulk SFs are often directly compared with ARPES spectra 36 , thus ignoring the role of the final states and the effect of the surface. The surface effects in SCES were considered in Refs. 39 43, and their influence on ARPES was discussed on a qualitative level. Thus, the formulation of the one-step approach in the localized basis is highly desirable as it would enable a quantitative comparison of many-body calculations results with the state-of-the-art ARPES data 44 .

According to the classification of Ref. 45, the strongly 
correlated transition metal compounds may be divided into two categories depending on the relation between Coulomb interaction $U$ within the $d$-shell and the charge transfer energy $\Delta$ between the metal ion and surrounding anions. In the Mott-Hubbard systems, $\Delta \gg U$, the valence band may be described by a one-band Hubbardtype mode $\mathrm{I}^{\sqrt{35}}$, while for charge-transfer systems, $\Delta \ll U$, an explicit account of the anion states is necessary $\underline{46}$.

In this paper, we formulate the one-step approach in the localized basis (Sec. II), and consider its application to Mott-Hubbard systems. In Sec. III] starting from the bulk Green's function (GF), we derive the GF of the semi-infinite system. After a short discussion of ARPES of layered systems in Sec. IIIA, we find an analytical formula for the photocurrent from a system with tangible dispersion in the direction normal to the surface (Sec. IV]. In Sec. V], we discuss how the formula reflects the role of final states and of the surface and give some illustration of its application. Technical details of the derivation are given in the Appendix.

\section{ARPES CALCULATION FOR LOCALIZED BASIS}

In this section we revisit the one-step theory of photoemission in order to formulate it in the Wannier representation for initial states. The localized basis is ideally suited for the electronic structure of SCES, and at a certain level of approximation it allows an elegant inclusion of surface effects.

\section{A. Sudden approximation}

We consider a semi-infinite crystal that extends over the half-space $z \leq z_{0}$, with a perfectly flat surface. The solid is irradiated with light given by the vector potential $\mathbf{A}(\mathbf{x}, t)=\mathbf{A}(\mathbf{x}) \cos \Omega t$ (we choose the gauge in which the scalar potential is zero). Within the sudden approximation ${ }^{12}$, the interaction between the excited electron and the photohole is neglected. Then the descriptions of the initial state and of the final state can be separated from each other. The steady radial photocurrent $j(\hat{q}, E)$ of electrons emerging from the solid along the observation direction defined by the unit vector $\hat{q}$ with energies between $E$ and $E+d E$ is then given

$$
\begin{aligned}
\text { by } & \\
j(\hat{q}, E) & =\frac{1}{2 \pi} \lim _{\substack{\mathbf{X}^{\prime} \rightarrow \mathbf{x} \\
\mathbf{X} \rightarrow \infty}}\left(\frac{\partial}{\partial X^{\prime}}-\frac{\partial}{\partial X}\right) \\
& \times \iint d^{3} \mathbf{x}_{1} d^{3} \mathbf{x}_{2} G\left(\mathbf{X}, \mathbf{x}_{1}, E\right) \hat{O}\left(\mathbf{x}_{1}\right) \\
& \times G^{+}\left(\mathbf{x}_{1}, \mathbf{x}_{2}, E-\hbar \Omega\right) \hat{O}\left(\mathbf{x}_{2}\right) G^{*}\left(\mathbf{x}_{2}, \mathbf{X}^{\prime}, E\right)
\end{aligned}
$$

where the vector $\mathbf{X}=X \hat{q}$ points in the direction of the detector, and $G(\mathbf{X}, \mathbf{x}, E)$ is the retarded propagator of the outgoing electron,

$$
G\left(\mathbf{x}, \mathbf{x}^{\prime}, \omega\right)=\left\langle\left\langle\hat{\psi}(\mathbf{x}) \mid \hat{\psi}^{\dagger}\left(\mathbf{x}^{\prime}\right)\right\rangle\right\rangle_{\omega} .
$$

In general, the anticommutator two-time retarded GF of two operators $\hat{A}$ and $\hat{B}$ is defined as

$$
\langle\langle\hat{A} \mid \hat{B}\rangle\rangle_{\omega} \equiv-i \int_{0}^{\infty}\langle\{\hat{A}(t), \hat{B}(0)\}\rangle e^{i \omega t} d t
$$

where $\{\hat{A}, \hat{B}\} \equiv \hat{A} \hat{B}+\hat{B} \hat{A}$, the time-dependent operator $\hat{A}(t)$ is $\hat{A}(t)=\exp (i t \hat{H}) \hat{A} \exp (-i t \hat{H})$, and the angular brackets denote the thermodynamic average $\langle\hat{A}\rangle \equiv \operatorname{Tr}[\exp (-\beta \hat{H}) \hat{A}] / \operatorname{Tr}[\exp (-\beta \hat{H})]$. The operator $\psi(\mathbf{x})$ annihilates an electron at the point $\mathbf{x}$, and $G^{+}\left(\mathbf{x}_{1}, \mathbf{x}_{2}, \omega\right)$ is the "lesser" function ${ }^{15 \mid 16}$ for the initial state

$$
G^{+}\left(\mathbf{x}_{1}, \mathbf{x}_{2}, \omega\right) \equiv-2 i f(E+\Phi) G^{\prime \prime}\left(\mathbf{x}_{1}, \mathbf{x}_{2}, \omega\right),
$$

where $\Phi$ is the work function, the vacuum level is at $E=0$, and $f(\omega)=1 /\left(e^{\beta \omega}+1\right)$ is the Fermi distribution function. We consider a non-magnetic solid and drop the spin index. Throughout the text the double prime denotes the imaginary part of a complex value, e.g., $G^{\prime \prime} \equiv$ $\operatorname{Im} G$. The operator $\hat{O}(\mathbf{x})=\frac{1}{2 c}[\mathbf{A}(\mathbf{x}) \cdot \mathbf{P}+\mathbf{P} \cdot \mathbf{A}(\mathbf{x})]$ is the electron-light coupling, with $\mathbf{P}=-i \nabla$ being the electron momentum operator and $\mathrm{c}$ the light velocity. The atomic units $\hbar=e=m_{e}=1$ are used. In resonant photoemission $\frac{47 \sqrt[50]{50}}{5}$ or in the presence of microscopic fields due to the dielectric screening 51152 the operator $\hat{O}$ is more involved, which complicates the calculation of matrix elements $\mathrm{M}\left(\mathbf{k}_{\|}, E\right)$ in Eq. 20p, but the theory presented below remains fully applicable

Following Ref. [16, we use the asymptotic formula for $G(\mathbf{X}, \mathbf{x}, E)$,

$$
G(\mathbf{X}, \mathbf{x}, E) \underset{\mathbf{X} \rightarrow \infty}{\longrightarrow} \frac{1}{2 \pi} \frac{\exp (i X \sqrt{2 E})}{X} \varphi_{>}(\mathbf{x}, \hat{q}, E)
$$

where $\varphi_{>}(\mathbf{x}, \hat{q}, E)$ is the LEED wave function. The inelastic scattering due to electron-electron interaction in the propagation of the outgoing electron may be taken into account phenomenologically by introducing an absorbing optical potential into the effective Schrödinger equation for the function $\varphi_{>}(\mathbf{x}, \hat{q}, E)^{53}[55$. Thereby the LEED function becomes a superposition of evanescent Bloch waves. Substitution of (3) and (2) into Eq. (1) gives 


$$
\begin{aligned}
j(\hat{q}, E) & =-\left(\frac{1}{2 \pi}\right)^{3}\left[\frac{2 f(E+\Phi) \sqrt{2 E}}{X^{2}}\right] \\
& \times \iint d^{3} \mathbf{x}_{1} d^{3} \mathbf{x}_{2} \varphi_{>}\left(\mathbf{x}_{1}, \hat{q}, E\right) \hat{O}\left(\mathbf{x}_{1}\right) G^{\prime \prime}\left(\mathbf{x}_{1}, \mathbf{x}_{2}, E-\hbar \Omega\right) \hat{O}\left(\mathbf{x}_{2}\right) \varphi_{>}^{*}\left(\mathbf{x}_{2}, \hat{q}, E\right) .
\end{aligned}
$$

Note that the initial states are confined inside the solid, so that the integration over $\mathbf{x}_{1}$ and $\mathbf{x}_{2}$ in (4) is essentially restricted to the crystal half-space, $\mathbf{x}_{i} \subset \mathcal{S}$, i.e.,

$$
\int_{\mathbf{x} \subset \mathcal{S}} d^{3} \mathbf{x}_{1} \ldots \equiv \iint_{-\infty}^{\infty} d x d y \int_{-\infty}^{z_{0}+\Delta z} d z \ldots
$$

which assumes that initial states vanish at a distance $\Delta z$ from the surface. With this in mind, and using the symmetry relation

$$
G\left(\mathbf{x}_{1}, \mathbf{x}_{2}, \omega\right)=G\left(\mathbf{x}_{2}, \mathbf{x}_{1}, \omega\right),
$$

we make an important next step (the details are given in the Appendix), and rewrite (4) in the form

$$
\begin{aligned}
j(\hat{q}, E) & =\left[\frac{f(E+\Phi) \sqrt{2 E}}{(2 \pi X)^{2}}\right] \mathcal{A}(\hat{q}, E-\hbar \Omega), \\
\mathcal{A}(\hat{q}, \omega) & =-\frac{1}{\pi} \operatorname{Im} \mathcal{G}(\hat{q}, \omega+i 0), \\
\mathcal{G}(\hat{q}, \omega) & =\left\langle\left\langle\hat{C} \mid \hat{C}^{\dagger}\right\rangle\right\rangle_{\omega},
\end{aligned}
$$

where the operator

$$
\hat{C}^{\dagger}(\hat{q}, E) \equiv \int d^{3} \mathbf{x} \hat{\psi}^{\dagger}(\mathbf{x}) \chi(\mathbf{x}, \hat{q}, E)
$$

creates an electron in a state with the wave function

$$
\begin{aligned}
\chi(\mathbf{x}, \hat{q}, E) & =\hat{O}(\mathbf{x}) \varphi_{>}^{*}(\mathbf{x}, \hat{q}, E), \mathbf{x} \subset \mathcal{S} \\
& =0, \text { otherwise }
\end{aligned}
$$

Equation (7) gives an explicit form of the SF to be calculated to obtain the photocurrent.

\section{B. Non-interacting electrons}

In a mean-field approach, the initial states are described by an effective one-particle Hamiltonian. In the basis of its eigenfunctions $\Psi_{i}(\mathbf{x})$, it reads

$$
\hat{H}_{\mathrm{mf}}=\sum_{i} E_{i} a_{i}^{\dagger} a_{i}
$$

where index $i$ incorporates all quantum numbers that define a quantum state of the system, $E_{i}$ being its energy. Then the electron annihilation operator is $\hat{\psi}(\mathbf{r})=$ $\sum_{\text {is }} \Psi_{i}(\mathbf{x}) a_{i}$, and the operator conjugate to $\hat{C}^{\dagger}$ of Eq. 9

$$
\begin{aligned}
\hat{C}(\hat{q}, E) & =\sum_{i} M_{i} a_{i}, \\
M_{i} & =\int_{\mathbf{x} \subset \mathcal{S}} d^{3} \mathbf{x} \varphi_{>}(\mathbf{x}, \hat{q}, E) \hat{O}(\mathbf{x}) \Psi_{i}(\mathbf{x}) \\
& =\int d^{3} \mathbf{x} \chi^{*}(\mathbf{x}, \hat{q}, E) \Psi_{i}(\mathbf{x}) .
\end{aligned}
$$

The last integration may be extended over the whole space, as both functions $\chi$ and $\Psi$ are confined inside the solid. The GF of Eq. (8) that defines the photocurrent is

$$
\mathcal{G}(\hat{q}, \omega)=\sum_{i} M_{i} M_{j}^{*}\left\langle\left\langle a_{i} \mid a_{j}^{\dagger}\right\rangle\right\rangle_{\omega} .
$$

The GFs in the right-hand side are trivially calculated

$$
G_{i j}(\omega) \equiv\left\langle\left\langle a_{i} \mid a_{j}^{\dagger}\right\rangle\right\rangle_{\omega}=\frac{\delta_{i j}}{\omega-E_{i}} .
$$

We see that the SF (7) reduces to the density of states (DOS) projected on the function $\chi(\mathbf{x}, \hat{q}, E)$

$$
\mathcal{A}(\hat{q}, \omega)=\sum_{i}\left|M_{i}\right|^{2} \delta\left(\omega-E_{i}\right) .
$$

Substituting Eq. (13) into Eq. (6), we recover the wellknown expression for the photocurrent in the mean-field one-step approach 13 19156/57. Note that the Hamiltonian (11) describes a semi-infinite crystal, which makes the calculation of the eigenvalues $E_{i}$ and eigenfunctions $\Psi_{i}(\mathbf{x})$ highly non-trivial even in the mean-field approximation.

\section{Interacting electrons}

For the non-interacting systems, the photoexcitation of an electron from a single-determinant $N$-electron eigenstate of the Hamiltonian (11) creates an $(N-1)$-electron eigenstate of the same Hamiltonian. The electronelectron interaction complicates the picture of the photoexcitation. On the mean-field level, the removal of an electron from an $N$-electron system changes the meanfield, but these changes are negligible for a macroscopic number of electrons. More important is the interaction beyond the mean-field: the two-particle (four-fermion operator) terms in the Hamiltonian, which account for the 
residual interaction, i.e., are the part of the bare Coulomb interaction responsible for the correlations in the electron motion ${ }^{5}$. In contrast to the bare Coulomb interaction, the residual interaction is a short-ranged one. It is often introduced on the model level via Hubbard-like terms, which are conveniently represented in the localized basis of Wannier functions 35 .

In SCES, the Hubbard terms are comparable with matrix elements of kinetic energy. This makes it impossible to present an $N$-electron eigenstate as a single determinant. The removal/addition of an electron from/to this state produces an $(N-1)-/(N+1)$-electron state that is a combination of a large number of eigenstates with different energies. As a consequence, the GF describing electron removal and additional spectra does not have the simple pole form of Eq. (12) but acquires a complex self-energy in the denominator. As a result, the SF $-G_{i i}^{\prime \prime}(\omega+i 0) / \pi$ is not anymore a single $\delta$-function, but it may have humps that come from the branch cut singularities of the self-energy, and that are called incoherent bands. These bands coming from the self-energy of the initial states are observed in ARPES as "satellites" that appear at binding energies different from the energies of "main peaks" of the mean field theory. For a proper interpretation of the experiment, the many-body GF describing the initial states should be incorporated into the one-step approach.

Note that the only approximation we have used to derive Eq. $(6)$ is the sudden approximation, and that Eq. (6) is fully general and applicable for a wide range of systems including strongly correlated systems. The role of the final state $\varphi_{>}^{*}(\mathbf{x}, \hat{q}, E)$ (the time reversed LEED state) is clear from Eqs. (9) and (10): it defines the form of the operator $\hat{C}_{\sigma}(\hat{q}, E)$, and, thus, the $\left.\mathrm{SF} \sqrt{7}\right)$, which is our ultimate aim. Thus, the angular and energy dependence of the photocurrent cannot be understood solely from the structure of the initial states. On the one hand, this complicates the interpretation of ARPES experiments, but, on the other hand, it allows to learn about final states from the measured spectra ${ }^{58}$.

We assume that the target crystal has two-dimensional (2D) lattice periodicity. Inside the solid, the time reversed LEED function may be written as

$$
\varphi_{>}^{*}(\mathbf{x}, \hat{q}, E)=e^{i \mathbf{k}_{\|} \mathbf{x}_{\|} U}\left(\mathbf{x}_{\|}, z, \hat{q}, E\right),
$$

where $\mathbf{x}_{\|}$is the radius-vector component parallel to the surface, $\mathbf{x}=\mathbf{x}_{\|}+z \mathbf{n}$, with $\mathbf{n}$ being the unity vector normal to the surface. The surface-parallel momentum component $\mathbf{q}_{\|}=\mathbf{k}_{\|}+\mathbf{G}_{\|}$is the sum of the momentum vector in the first Brillouin zone $\mathbf{k}_{\|}$and $2 \mathrm{D}$ reciprocal lattice vector $\mathbf{G}_{\|}$. The function $U\left(\mathbf{x}_{\|}, z, \hat{q}, E\right)$ is periodic in $\mathbf{x}_{\|}$and may be written as a combination of evanescent waves [cf. Eq. (37) of Ref. 16]:

$$
\begin{aligned}
& U\left(\mathbf{x}_{\|}, z, \hat{q}, E\right)=\sum_{m} \varphi_{m}^{*}\left(\mathbf{x}, \mathbf{k}_{\|}, E\right), \\
& \varphi_{m}^{*}\left(\mathbf{x}, \mathbf{k}_{\|}, E\right) \equiv e^{i k_{\perp, m}\left(z-z_{0}\right)} u_{m}\left(\mathbf{x}, \mathbf{k}_{\|}, E\right),
\end{aligned}
$$

where $m$ is the band index and $k_{\perp, m}\left(E, \mathbf{k}_{\|}\right)=k_{z, m}^{\prime}-$ $i k_{z, m}^{\prime \prime}\left(k_{z, m}^{\prime}, k_{z, m}^{\prime \prime}>0\right)$ is the complex momentum component in the direction perpendicular to the surface. The functions $u_{m}$ have the periodicity of the 3D crystal, with the Bravais lattice vectors $\mathbf{R}=\mathbf{R}_{\|}+l c \mathbf{n}$, where $l \leq z_{0} / c$ is an integer and $c$ is the lattice period in $z$ direction. For both $\mathbf{x}$ and $\mathbf{x}+\mathbf{R}$ inside the solid it is $u_{m}\left(\mathbf{x}+\mathbf{R}, \mathbf{k}_{\|}, E\right)=u_{m}\left(\mathbf{x}, \mathbf{k}_{\|}, E\right)$. Inside the crystal, the function produced by the perturbation $\hat{O}$ acting on the final state $\varphi_{>}^{*}$ in Eq. 10 is, clearly, also a combination of evanescent waves

$$
\begin{aligned}
\chi(\mathbf{x}, \hat{q}, E) & =\sum_{m} \chi_{m}\left(\mathbf{x}, \mathbf{k}_{\|}, E\right) \\
\chi_{m}\left(\mathbf{x}, \mathbf{k}_{\|}, E\right) & =\hat{O}(\mathbf{x}) e^{i \mathbf{k}_{\|} \mathbf{x}_{\|}} \varphi_{m}^{*}\left(\mathbf{x}, \mathbf{k}_{\|}, E\right) .
\end{aligned}
$$

Thus, the function $\chi(\mathbf{x}, \hat{q}, E)$ is localized at the surface, which reflects the surface sensitivity of the photoemission spectroscopy. If one of the waves dominates the sum in Eq. 15 its localization can be expressed by the "inelastic mean free path" $L \sim 1 / 2 k_{z}^{\prime \prime}\left(E, \mathbf{k}_{\|}\right)$.

In order to proceed further, we chose a basis of localized functions suitable for the description of the initial state. For example, it may be the basis of Wannier functions for a set of bands within some energy window around $\omega=E-\hbar \Omega$. We write the electron annihilation operator for the initial state in the form

$$
\hat{\psi}(\mathbf{r})=\sum_{\mathbf{R}, \alpha} w_{\alpha}(\mathbf{r}-\mathbf{R}-\mathbf{s}) a_{\mathbf{R}, \alpha}
$$

where $a_{\mathbf{R}, \alpha}$ annihilates an electron in the state $w_{\alpha}(\mathbf{r}-$ $\mathbf{R}-\mathbf{s}$ ) localized at the lattice site $\mathbf{R}+\mathbf{s}$, where $\mathbf{s}$ is a basis vector of the unit cell, and $\alpha$ accumulates $\mathbf{s}$ and all the relevant quantum numbers. For the operator $\hat{C}$ of Eq. (9) we obtain (see Appendix) 


$$
\begin{aligned}
\hat{C}(\hat{q}, E) & =\sqrt{N_{\|}} \sum_{l, \mathbf{s}, \alpha} \int d z \iint d^{2} \mathbf{x}_{\|} \varphi_{>}(\mathbf{x}, \hat{q}, E) \hat{O}(\mathbf{x}) w_{\alpha}\left[\mathbf{x}_{\|}+(z-l c) \mathbf{n}-\mathbf{s}\right] a_{\mathbf{k}_{\|}, l, \alpha} \\
& =\sqrt{N_{\|}} \sum_{m, \alpha} \mathbf{M}_{m, \alpha}\left(\mathbf{k}_{\|}, E\right) \sum_{l} e^{-i k_{\perp, m}^{*}\left(l c-z_{0}\right)} a_{\mathbf{k}_{\|}, l, \alpha}, \\
& =\sqrt{\frac{N_{\|}}{N_{\perp}}} \sum_{m, \alpha} \mathbf{M}_{m, \alpha}\left(\mathbf{k}_{\|}, E\right) \sum_{p} e^{i p z_{0}} \Delta_{m, p} a_{\mathbf{k}_{\|}, p, \alpha}, \\
\mathbf{M}_{m, \alpha}\left(\mathbf{k}_{\|}, E\right) & \equiv \int d^{3} \mathbf{x} \chi_{m}^{*}\left(\mathbf{x}, \mathbf{k}_{\|}, E\right) w_{\alpha}\left[\mathbf{x}-z_{0} \mathbf{n}-\mathbf{s}\right], \\
\Delta_{m, p} & \equiv \sum_{l=-\infty}^{z_{0} / c} e^{-i\left(k_{\perp, m}^{*}-p\right)\left(l c-z_{0}\right)}=\left\{1-e^{i\left(k_{\perp, m}^{*}-p\right) c}\right\}^{-1},
\end{aligned}
$$

where we have introduced the Fourier transforms

$$
\begin{aligned}
a_{\mathbf{k}_{\|}, l, \alpha} & =\frac{1}{\sqrt{N_{\|}}} \sum_{\mathbf{R}_{\|}} \mathrm{e}^{-i \mathbf{k}_{\|} \mathbf{R}_{\|}} a_{\mathbf{R}_{\|,}, l, \alpha}, \\
a_{\mathbf{k}, \alpha} & =a_{\mathbf{k}_{\|}, p, \alpha}=\frac{1}{\sqrt{N_{\perp}}} \sum_{l=-\infty}^{\infty} \mathrm{e}^{-i p l c} a_{\mathbf{k}_{\|}, l, \alpha} .
\end{aligned}
$$

Here $N_{\|}$is the number of sites in the plane, $N_{\perp}$ is the number of planes in the system, and $\mathbf{k}=\mathbf{k}_{\|}+p \mathbf{n}$. Operator $a_{\mathbf{k}_{\|}, l, \alpha}$ annihilates an electron in a layer Bloch state

$$
\begin{aligned}
& w_{\mathbf{k}_{\|}, \alpha}(\mathbf{r}-l c \mathbf{n})= \\
& \frac{1}{\sqrt{N_{\|}}} \sum_{\mathbf{R}_{\|}} \mathrm{e}^{i \mathbf{k}_{\|} \mathbf{R}_{\|}} w_{\alpha}\left[\mathbf{x}_{\|}-\mathbf{R}_{\|}+(z-l c) \mathbf{n}-\mathbf{s}\right],
\end{aligned}
$$

localized at $l$-th layer, while $a_{\mathbf{k}, \mathbf{s}, \alpha}$ annihilates an electron in a bulk Bloch state

$$
\begin{aligned}
& w_{\mathbf{k}, \alpha}(\mathbf{r})=\frac{1}{\sqrt{N_{\perp}}} \sum_{l} \mathrm{e}^{i p l c} w_{\mathbf{k}_{\|}, \alpha}(\mathbf{r}-l c \mathbf{n}) \\
& =\frac{1}{\sqrt{N_{\|} N_{\perp}}} \sum_{\mathbf{R}} \mathrm{e}^{i \mathbf{k} \mathbf{R}} w_{\alpha}(\mathbf{r}-\mathbf{R}-\mathbf{s}) .
\end{aligned}
$$

Equation (17) expresses the conservation of the momentum parallel to the surface. The expression $\sqrt{19}$ for $\hat{C}$ shows that, generally, all states with different perpendicular momenta $p$ contribute to the photocurrent for a given $\mathbf{k}_{\|}$and $E$. In Ref. 16, it was pointed out that the factor $\Delta_{m, p}(21)$ is sharply peaked at $k_{\perp, m}^{\prime}=p$ if $k_{\perp, m}^{\prime \prime} c \ll 1$ [cf. Eqs. (42)-(47) of Ref. 16]. In this particular case, the crystal momentum is conserved also in $z$ direction. In Sec. IV we will return to this discussion.

Equations (18) and (19) allow to write the GF of
Eq. (8) in the form

$$
\begin{aligned}
& \mathcal{G}(\hat{q}, \omega)=\frac{N_{\|}}{N_{\perp}} \sum_{m_{1}, m_{2}, \backslash \alpha_{1}, \alpha_{2}} \mathrm{M}_{m_{1}, \alpha_{1}} \mathrm{M}_{m_{2}, \alpha_{2}}^{*} \\
& \times \sum_{l_{1}, l_{2}} e^{i k_{\perp, m_{2}}\left(l_{2} c-z_{0}\right)-i k_{\perp, m_{1}}^{*}\left(l_{1} c-z_{0}\right)} G_{\mathbf{k}_{\|}, l_{1}, l_{2}, \alpha_{1}, \alpha_{2}}(\omega) \\
& =\frac{N_{\|}}{N_{\perp}} \sum_{m_{1}, m_{2}, \backslash \alpha_{1}, \alpha_{2}} \mathrm{M}_{m_{1}, \alpha_{1}} \mathrm{M}_{m_{2}, \alpha_{2}}^{*} \\
& \times \sum_{p_{1}, p_{2}} e^{i\left(p_{1}-p_{2}\right) z_{0}} \Delta_{m_{1}, p_{1}} \Delta_{m_{2}, p_{2}}^{*} G_{\mathbf{k}_{\|}, p_{1}, p_{2}, \alpha_{1}, \alpha_{2}}(\omega), \\
& G_{\mathbf{k}_{\|}, l_{1}, l_{2}, \alpha_{1}, \alpha_{2}}(\omega) \equiv\left\langle\left\langle a_{\mathbf{k}_{\|}, l_{1}, \alpha_{1}} \mid a_{\mathbf{k}_{\|}, l_{2}, \alpha_{2}}^{\dagger}\right\rangle\right\rangle_{\omega}
\end{aligned}
$$

The GF of semi-infinite crystal

$$
G_{\mathbf{k}_{\|}, p_{1}, p_{2}, \alpha_{1}, \alpha_{2}}(\omega) \equiv\left\langle\left\langle a_{\mathbf{k}_{\|}, p_{1}, \alpha_{1}} \mid a_{\mathbf{k}_{\|}, p_{2}, \alpha_{2}}^{\dagger}\right\rangle\right\rangle_{\omega}
$$

depends on the pair of perpendicular momenta because of the broken translational invariance in the surface-normal direction.

\section{SEMI-INFINITE MOTT-HUBBARD SYSTEM}

Now we consider a system whose valence band spectrum may be described by an effective one-band Hamiltonian $\hat{H}_{\text {eff }}$ on a Bravais lattice, i.e., we have only one sort of orbitals $\phi(\mathbf{r}-\mathbf{R})$ at the lattice sites $\mathbf{R}=\mathbf{R}_{\|}+z \mathbf{n}$. For an infinite crystal, the GF is diagonal in the $\mathbf{k}$-space

$$
\begin{aligned}
\left\langle\left\langle a_{\mathbf{k}_{1}} \mid a_{\mathbf{k}_{\mathbf{2}}}^{\dagger}\right\rangle\right\rangle_{\omega} & =\delta_{\mathbf{k}_{1}, \mathbf{k}_{2}} G_{b, \mathbf{k}}(\omega), \\
G_{b, \mathbf{k}}(\omega) & =\frac{1}{\omega-\varepsilon_{\mathbf{k}}-\Sigma_{\mathbf{k}, \omega}},
\end{aligned}
$$


where $\mathbf{k}=\mathbf{k}_{\|}+p \mathbf{n}$. Here we do not specify the Hamiltonian $\hat{H}_{\text {eff }}$ but assume only that the mean-field energy $\varepsilon_{\mathbf{k}}$ and the self-energy $\Sigma_{\mathbf{k}, \omega}$ may be calculated for the bulk system with the full account of many-body effects. The momentum-dependent SF

$$
A_{\mathrm{b}}(\mathbf{k}, \omega+i 0)=-\operatorname{Im} G_{b, \mathbf{k}}(\omega+i 0) / \pi
$$

is the main characteristic of the electronic structure of SCES. It contains information both about the quasiparticle energy dispersion and about the incoherent bands.

\section{A. ARPES from a layered system}

Many systems of current interest are built of weakly coupled layers or chains: High-Tc cuprates and Febased superconductors, quasi-one-dimensional magnetic systems, ruthenites, iridates, etc. If the surface coincides with the two-dimensional layer or is built of onedimensional (1D) chains we can neglect the dispersion in the surface-normal direction. Then the planes become decoupled, and the GF of Eq. 277, does not depend on $l_{1}$ and $l_{2}$. For the Mott-Hubbard system we can write $G_{\mathbf{k}_{\|}, l_{1}, l_{2}, \alpha_{1}, \alpha_{2}}(\omega)=\delta_{l_{1}, l_{2}} G_{b, \mathbf{k}_{\|}}(\omega)$. Equation 25 then yields

$$
\begin{aligned}
& \mathcal{G}(\hat{q}, \omega)=\left\langle\left\langle\hat{C}_{\sigma} \mid \hat{C}_{\sigma}^{\dagger}\right\rangle\right\rangle_{\omega} \propto G_{b, \mathbf{k}_{\|}}(\omega), \\
& \mathcal{A}(\hat{q}, \omega) \propto A_{b}(\mathbf{k}, \omega)
\end{aligned}
$$

Thus, for systems with a negligible dispersion normal to the surface ARPES directly measures the SF of the electron GF.

\section{B. Account of the surface in a 3D system}

However, the actual crystals are three-dimensional. Even in quasi-1D or quasi-2D systems the chains or the layers are coupled, and the energy of the photohole disperses with $k_{\perp}$. This dispersion may be small compared with the dispersion parallel to the surface, but with the progress in angular and energy resolution $\frac{44}{4}$ it becomes measurable, which calls for a more thorough theoretical analysis of the surface-normal degree of freedom, which is proposed below.

In the equation of motion for the GF

$$
\omega\left\langle\left\langle a_{\mathbf{k}_{1}} \mid a_{\mathbf{k}_{2}}^{\dagger}\right\rangle\right\rangle_{\omega}=\delta_{\mathbf{k}_{1}, \mathbf{k}_{2}}+\left(\varepsilon_{\mathbf{k}}+\Sigma_{\mathbf{k}, \omega}\right)\left\langle\left\langle a_{\mathbf{k}_{1}} \mid a_{\mathbf{k}_{\mathbf{2}}}^{\dagger}\right\rangle\right\rangle_{\omega},
$$

which straightforwardly follows from Eqs. 29 and 30 , we perform in both sides the Fourier transform $a_{\mathbf{k}_{\|}, l}=$ $\left(1 / \sqrt{N_{\perp}}\right) \sum_{p} \mathrm{e}^{i p l c} a_{\mathbf{k}}$ inverse to 23 and obtain the equation of motion for the "interlayer" GF of Eq. (27)

$$
\begin{aligned}
\omega G_{\mathbf{k}_{\|}, l_{1}, l_{2}}(\omega) & =\delta_{l_{1}, l_{2}}+\sum_{l} h_{l_{1}, l}\left(\mathbf{k}_{\|}, \omega\right) G_{\mathbf{k}_{\|}, l, l_{2}}(\omega), \\
h_{l, l_{2}}\left(\mathbf{k}_{\|}, \omega\right) & \equiv \frac{1}{N_{\perp}} \sum_{p} e^{i p\left(l-l_{2}\right) c}\left(\varepsilon_{\mathbf{k}}+\Sigma_{\mathbf{k}, \omega}\right)
\end{aligned}
$$

Equation (32) has the form of an equation of motion for an effective 1D tight-binding system with an energydependent (and generally non-Hermitian) Hamiltonian

$$
\hat{h}\left(\mathbf{k}_{\|}, \omega\right)=\sum_{l_{1}, l_{2}} h_{l_{1}, l_{2}}\left(\mathbf{k}_{\|}, \omega\right) a_{\mathbf{k}_{\|}, l_{1}}^{\dagger} a_{\mathbf{k}_{\|}, l_{2}}
$$

with hopping amplitudes given by Eq. (33).

Now we proceed with a semi-infinite crystal. The surface may be introduced as a perturbation $\hat{V}$ that breaks an infinite crystal into two non-interacting parts. In Refs. 41 and 59 the coupling between the two parts is eliminated by means of a non-diagonal perturbation $V_{l, l_{2}}=-h_{l, l_{2}}$. We achieve the same result using the diagonal perturbation of the form

$$
\hat{V}=\varepsilon_{0} \sum_{i} a_{\mathbf{k}_{\|}, i}^{\dagger} a_{\mathbf{k}_{\|}, i}
$$

where $i$ enumerates the atomic planes of a slab that divides the crystal into two semi-infinite parts. The width of the slab should be equal or larger than the maximal distance $\left(l-l_{2}\right) c$ for which the hopping integrals $h_{l, l_{2}}$ in Eq. (32) are non-zero. In the limit $\varepsilon_{0} \rightarrow \infty$ the two half-spaces are separated by an infinite barrier. Similar approaches are used for the description of vacancies ${ }^{60}$, in the cavity method of DMFT ${ }^{25}$, and for the hard-core constraint for magnon pairs in acute-angle helimagnets 61162 (the bound states of magnons being analogues of the surface states).

Note that the perturbation $\hat{V}$ leads to a relaxation of the system, which changes the effective Hamiltonian $\hat{h}\left(\mathbf{k}_{\|}, \omega\right)$. These changes are expected to be localized at the surface and, in principle, can be taken into account in a self-consistent way. Here we neglect it and consider the simplest case when only adjacent planes are coupled by $\hat{h}\left(\mathbf{k}_{\|}, \omega\right)$

$$
\begin{aligned}
\varepsilon_{\mathbf{k}} & =\varepsilon_{\mathbf{k}_{\|}}-2 t_{\mathbf{k}_{\|}} \cos p c, \\
\Sigma_{\mathbf{k}, \omega} & =\Sigma_{\mathbf{k}_{\|}, \omega}-2 \tau_{\mathbf{k}_{\|}, \omega} \cos p c,
\end{aligned}
$$

$c$ being the inter-plane distance. Then we may retain in Eq. (35) only the term with $z_{i}=0$. The assumption (36) is natural for a narrow-band system, and the local character of the self-energy (37) is also a commonly accepted approximation 25126138 . Note that we do not make any as-

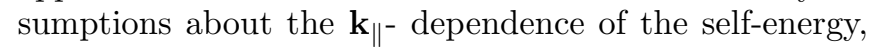
which may be quite strong $38 \mid 63 \sqrt[65]{6}$. We then obtain the bulk GF of Eq. [30] in the form

$$
G_{b, \mathbf{k}}(\omega)=\left[\omega-\sigma_{\mathbf{k}_{\|}, \omega}+2 T_{\mathbf{k}_{\|}, \omega} \cos p c\right]^{-1},
$$

where we have included the dispersion parallel to the surface $\varepsilon_{\mathbf{k}_{\|}}$into the real part of the self-energy: $\sigma_{\mathbf{k}_{\|}, \omega} \equiv$ $\varepsilon_{\mathbf{k}_{\|}}+\Sigma_{\mathbf{k}_{\|}, \omega}$ and $T_{\mathbf{k}_{\|}, \omega} \equiv t_{\mathbf{k}_{\|}}+\tau_{\mathbf{k}_{\|}, \omega}$.

The equation of motion for the GF of the perturbed system then reads

$$
\begin{aligned}
\omega G_{\mathbf{k}_{\|}, l_{1}, l_{2}} & =\delta_{l_{1}, l_{2}}+\left(\varepsilon_{\mathbf{k}_{\|}}+\Sigma_{\mathbf{k}_{\|}, \omega}\right) G_{\mathbf{k}_{\|}, l_{1}, l_{2}} \\
& -T_{\mathbf{k}_{\|}, \omega}\left(G_{\mathbf{k}_{\|}, l_{1}+1, l_{2}}+G_{\mathbf{k}_{\|}, l_{1}-1, l_{2}}\right) \\
& +\delta_{l_{1}, 0} \varepsilon_{0} G_{\mathbf{k}_{\|}, 0, l_{2}}
\end{aligned}
$$


We perform the double Fourier transform

$$
G_{\mathbf{k}_{\|}, p_{1}, p_{2}}(\omega)=\frac{1}{N_{\perp}} \sum_{l, l^{\prime}} e^{-i\left(p_{1} l+i p_{2} l^{\prime}\right) c} G_{\mathbf{k}_{\|}, l, l^{\prime}}(\omega),
$$

in both sides of Eq. (39) to obtain for the GF of Eq. 28)

$$
G_{\mathbf{k}_{\|}, p_{1}, p_{2}}(\omega)=G_{b, \mathbf{k}_{1}}(\omega)\left(\delta_{p_{1}, p_{2}}+\frac{\varepsilon_{0}}{\sqrt{N_{\perp}}} G_{0}\right),
$$

where we have defined

$$
\mathrm{G}_{l} \equiv G_{\mathbf{k}_{\|}, l, p_{2}}=\frac{1}{\sqrt{N_{\perp}}} \sum_{p} e^{i p l c} G_{\mathbf{k}_{\|}, p, p_{2}} .
$$

Now we substitute (40) into the left hand side of Eq. (41) for $l=0$ and find

$$
\begin{aligned}
\mathrm{G}_{0} & =\frac{1}{\sqrt{N_{\perp}}} \frac{G_{b, \mathbf{k}_{\mathbf{2}}}(\omega)}{1-\varepsilon_{0} g_{\mathbf{k}_{\|}}(\omega)}, \\
g_{\mathbf{k}_{\|}}(\omega) & \equiv \frac{1}{N_{\perp}} \sum_{p} G_{b, \mathbf{k}}(\omega) .
\end{aligned}
$$

Finally, Eq. 40 gives the GF of the perturbed system

$$
\begin{aligned}
G_{\mathbf{k}_{\|}, p_{1}, p_{2}}(\omega) & =G_{b, \mathbf{k}_{2}}(\omega)\left\{\delta_{p_{1}, p_{2}}+\frac{\varepsilon_{0} G_{b, \mathbf{k}_{1}}(\omega)}{N_{\perp}\left[1-\varepsilon_{0} g_{\mathbf{k}_{\|}}(\omega)\right]}\right\} \\
& \underset{\varepsilon_{0} \rightarrow \infty}{\longrightarrow} G_{b, \mathbf{k}_{2}}(\omega)\left\{\delta_{p_{1}, p_{2}}-\frac{G_{b, \mathbf{k}_{1}}(\omega)}{N_{\perp} g_{\mathbf{k}_{\|}}(\omega)}\right\} .
\end{aligned}
$$

Thus, we have found the GF of the Hamiltonian $\hat{h}_{1}\left(\mathbf{k}_{\|}, \omega\right)=\hat{h}\left(\mathbf{k}_{\|}, \omega\right)+\varepsilon_{0} a_{\mathbf{k}_{\|}, 0}^{\dagger} a_{\mathbf{k}_{\|}, 0}$. Equation 44 is the desired result: it gives the GF for the semi-infinite crystal that extends over the half-space $z \leq z_{0}=-c$, which is necessary for the calculation of ARPES via Eqs. (6) and $(26)$.

Note that the approximations given by Eqs. (36) and (37) may be easily relaxed by using a thicker slab in Eq. (35). In this case, the GF may be found by successively applying this trick 60 : based on Eq. 43 we find the GF of the Hamiltonian $\hat{h}_{2}\left(\mathbf{k}_{\|}, \omega\right)=\hat{h}_{0}\left(\mathbf{k}_{\|}, \omega\right)+$ $\varepsilon_{0} a_{\mathbf{k}_{\|, c}^{\dagger}}^{\dagger} a_{\mathbf{k}_{\|}, c}$ with two perturbed planes and employ it to find the GF for three perturbed planes, etc.

A similar technique may be used to account for the surface relaxation of the system. In this case, the charge selfconsistency may require the diagonal terms $h_{l, l}\left(\mathbf{k}_{\|}, \omega\right)$ (33) to be $l$-dependent ${ }^{39 / 40}$, and also the non-diagonal terms $h_{l, l_{2}}\left(\mathbf{k}_{\|}, \omega\right)$ of the effective Hamiltonian (34) may depend on both indices $l$ and $l_{2}$ rather than on their difference. These deviations from the Hamiltonian (34) obtained from the bulk values of $\varepsilon_{\mathbf{k}}+\Sigma_{\mathbf{k}, \omega}$ are expected to have local character, and, thus, can be treated by Eqs. (39) - (43). Thereby, the problem is reduced to the problem of a few impurities in a 1D chain. These changes will perturb the electronic structure near the surface, and surface states may emerge. As mentioned above, the surface states that decouple from the bulk continuum have close analogy to the bound states of magnons in $1 \mathrm{D}$ magnets $\$$.

\section{ARPES FROM A 3D MOTT-HUBBARD SYSTEM}

In this section we consider the case when one of the waves dominates the sum in Eq. (16), so the timereversed LEED function $\varphi_{>}^{*}(\mathbf{x}, \hat{q}, E)(\overline{14})$ inside the solid may be approximated by a single evanescent wave:

$$
\varphi_{>}^{*}(\mathbf{x}, \hat{q}, E) \approx e^{i\left[\mathbf{k}_{\|} \mathbf{x}_{\|}+k_{\perp}\left(z-z_{0}\right)\right]} u\left(\mathbf{x}, \mathbf{k}_{\|}, E\right),
$$

where $k_{\perp}=k_{z}^{\prime}-i k_{z}^{\prime \prime}$. Then Eq. (26) acquires the form

$$
\begin{aligned}
\mathcal{G}(\hat{q}, \omega) & \equiv\left\langle\left\langle\hat{C}(\hat{q}, E) \mid \hat{C}^{\dagger}(\hat{q}, E)\right\rangle\right\rangle_{\omega}=\left|\mathrm{M}\left(\mathbf{k}_{\|}, E\right)\right|^{2} \times \\
& \times \frac{N_{\|}}{N_{\perp}} \sum_{p_{1}, p_{2}} e^{i\left(p_{1}-p_{2}\right) z_{0}} \Delta_{p_{1}} \Delta_{p_{2}}^{*} G_{\mathbf{k}_{\|}, p_{1}, p_{2}}(\omega),
\end{aligned}
$$

where we define $\omega \equiv E-\hbar \Omega$. Substituting the expression (44) for the GF of the semi-infinite system into Eq. (46) and setting there $z_{0}=-c$ (the surface layer) we have

$$
\mathcal{G}(\hat{q}, \omega)=\left|\mathrm{M}\left(\mathbf{k}_{\|}, E\right)\right|^{2} N_{\|}\left[I_{1}\left(\mathbf{k}_{\|}, \omega\right)-I_{2}\left(\mathbf{k}_{\|}, \omega\right)\right],
$$

where

$$
\begin{aligned}
I_{1}\left(\mathbf{k}_{\|}, \omega\right) & =\frac{1}{2 \pi c} \int_{-\pi / c}^{\pi / c}\left|\Delta_{p}\right|^{2} G_{b, \mathbf{k}}(\omega) d p, \\
I_{2}\left(\mathbf{k}_{\|}, \omega\right) & =\frac{1}{g_{\mathbf{k}_{\|}}(\omega)} I_{21}\left(k_{z}^{\prime}\right) I_{21}\left(-k_{z}^{\prime}\right), \\
I_{21}\left(k_{z}^{\prime}\right) & =\frac{1}{2 \pi c} \int_{-\pi / c}^{\pi / c} \Delta_{p} e^{-i p c} G_{b, \mathbf{k}}(\omega) d p .
\end{aligned}
$$

Here $G_{b, \mathbf{k}}(\omega)$ is given by Eq. 38 with $\mathbf{k}=\mathbf{k}_{\|}+p \mathbf{n}$, $\Delta_{p}=\left\{1-\exp \left[i\left(k_{z}^{\prime}-p\right)-k_{z}^{\prime \prime}\right] c\right\}^{-1}$. The integrand of $I_{1}$ is defined by the bulk GF of Eq. (30), and $I_{2}$ comes from the surface term of (44).

The integrals (48) and (50) are calculated using the residue theorem (see the details in Appendix)

$$
\begin{aligned}
I & =\int_{-\pi}^{\pi} R(\cos \varphi, \sin \varphi) d \varphi \\
& =\oint_{|z|=1} R_{0}(z) d z=2 \pi i \sum_{m=1}^{n} \operatorname{Res}_{z=z_{m}} R_{0}(z),
\end{aligned}
$$

where $R(u, v)$ is a rational function of $u$ and $v$, and $z_{m}$, $m=1, \ldots, n$ are poles of rational function $R_{0}(z)=$ $-\frac{i}{z} R\left[\frac{1}{2}\left(z+\frac{1}{z}\right), \frac{1}{2}\left(z-\frac{1}{z}\right)\right]$ that lie inside the circle $|z|<$ 1. We have two residues for $I_{1}$,

$$
I_{1}\left(\mathbf{k}_{\|}, \omega\right)=R_{1}\left(\mathbf{k}_{\|}, \omega\right)+R_{2}\left(\mathbf{k}_{\|}, \omega\right),
$$

which are given by Eqs. A10 and A11 and one residue for $I_{21}$ A12. The final expression is

$$
\mathcal{G}(\hat{q}, \omega)=K\left(\mathbf{k}_{\|}, E\right) F\left(\mathbf{k}, \omega-\sigma_{\mathbf{k}_{\|}, \omega}\right),
$$


where

$$
\begin{aligned}
K\left(\mathbf{k}_{\|}, E\right) & =\frac{\left|\mathrm{M}\left(\mathbf{k}_{\|}, E\right)\right|^{2} N_{\|}}{1-e^{-2 k^{\prime \prime} c}}, \\
F(\mathbf{k}, \epsilon) & =\frac{1}{\epsilon-\epsilon_{\mathbf{k}, \omega}-B_{\mathbf{k}, \omega}^{2} g_{s}\left(\epsilon, T_{\mathbf{k}_{\|}, \omega}\right)}, \\
\epsilon_{\mathbf{k}, \omega} & \equiv-2 T_{\mathbf{k}_{\|}, \omega} e^{-k_{z}^{\prime \prime} c} \cos k_{z}^{\prime} c \\
B_{\mathbf{k}, \omega}^{2} & \equiv T_{\mathbf{k}_{\|}, \omega}^{2}\left(1-e^{-2 k_{z}^{\prime \prime} c}\right),
\end{aligned}
$$

and the function

$$
\begin{aligned}
g_{s}(\epsilon, b) & \equiv\left\langle\left\langle a_{0} \mid a_{0}^{\dagger}\right\rangle\right\rangle_{\epsilon} \\
& =1 /\left\{\epsilon-b^{2} /\left[\epsilon-b^{2} /(\epsilon-\cdots)\right]\right\} \\
& =1 /\left(\epsilon-b^{2} g_{s}(\epsilon, b)\right) \\
& =\left\{\epsilon-\operatorname{sgn}[\operatorname{Re}(\epsilon)] \sqrt{\epsilon^{2}-4 b^{2}}\right\} / 2 b^{2}
\end{aligned}
$$

is the GF for the states localized at the edge of a semi-infinite chain described by the tight-binding Hamiltonian $\frac{6667}{h} \hat{h}=b \sum_{l \geq 0} a_{l}^{\dagger} a_{l+1}$.

Equation (55) represents the function $F(\mathbf{k}, \epsilon)$ in a continued-fraction form. This ensures the correct analytic properties of $\mathcal{G}(\hat{q}, \omega)$, see Eq. 47), as a function of complex energy $\epsilon=\omega-\sigma_{\mathbf{k}_{\|}, \omega}=\omega-\varepsilon_{\mathbf{k}_{\|}}-\Sigma_{\mathbf{k}_{\|}, \omega}$. The GF is an analytic function in the whole complex energy plane with the exception of the real axis, where it may have poles and branch cuts66. In the upper (lower) half-plane it coincides with the retarded (advanced) GF. It is easy to see that the function $F\left(\mathbf{k}, \omega-\sigma_{\mathbf{k}_{\|}, \omega}\right)$ coincides with the bulk GF of Eq. (38) in the so-called direct-transitions limit $k_{z}^{\prime \prime} \rightarrow 0$. In this limit it is $\epsilon_{\mathbf{k}, \omega} \rightarrow-2 T_{\mathbf{k}_{\|}, \omega} \cos k_{z}^{\prime} c$ and $B_{\mathbf{k}, \omega}^{2} \rightarrow 0$, and finally Eq. (54) becomes

$$
F(\mathbf{k}, \omega) \underset{k_{z}^{\prime \prime} \rightarrow 0}{\longrightarrow} G_{b, \mathbf{k}_{\|}+k_{z}^{\prime} \mathbf{n}}(\omega)
$$

A pole of the bulk GF $G_{b, \mathbf{k}_{\|}+k_{z}^{\prime} \mathbf{n}}(\omega)$ may occur if both $\Sigma_{\mathbf{k}, \omega}$ is real and the energy $\omega_{0}(\mathbf{k})$ satisfies the equation

$$
\omega_{0}(\mathbf{k})=\sigma_{\mathbf{k}_{\|}, \omega_{0}}-2 T_{\mathbf{k}_{\|}, \omega_{0}} \cos k_{z}^{\prime} c
$$

In the vicinity of this energy it is $G_{b, \mathbf{k}_{\|}+k_{z}^{\prime} \mathbf{n}}(\omega) \approx$ $Z_{\mathbf{k}}\left(\omega_{0}\right) /\left(\omega-\omega_{0}\right)$ with the residue $Z_{\mathbf{k}}(\omega)=$ $\left[1-\partial \Sigma_{\mathbf{k}, \omega} / \partial \omega\right]^{-1}$. For $k_{z}^{\prime \prime} c \ll 1$ the pole transforms into a resonance of a Lorentzian form

$$
\begin{aligned}
F_{r} & \approx \frac{Z_{\mathbf{k}}\left(\omega_{r}\right)\left(1+k_{z}^{\prime \prime} c\right)}{2 k_{z}^{\prime \prime} c\left[\omega-\omega_{r}+i \Gamma\right]}, \\
\Gamma & \approx 2 Z_{\mathbf{k}}\left(\omega_{r}\right)\left|T_{\mathbf{k}_{\|}, \omega_{r}}\right| k_{z}^{\prime \prime} c \sqrt{\left(k_{z}^{\prime \prime} c\right)^{2}+\sin ^{2} k_{z}^{\prime} c} \\
& \approx k_{z}^{\prime \prime} v_{h}, \quad v_{h} \equiv 2 Z_{\mathbf{k}}\left(\omega_{r}\right)\left|T_{\mathbf{k}_{\|}, \omega_{r}} \sin k_{z}^{\prime} c\right| c
\end{aligned}
$$

where the energy of the resonance satisfies the equation $\omega_{r}-\sigma_{\mathbf{k}_{\|}, \omega_{r}}=-2 T_{\mathbf{k}_{\|}, \omega_{r}} \cos k_{z}^{\prime} c / \cosh k_{z}^{\prime \prime} c$, which for a small decay index $k_{z}^{\prime \prime} c$ gives $\omega_{r}(\mathbf{k}) \approx \omega_{0}(\mathbf{k})$ Equation (61) is the well-known expression for the resonance width $\Gamma^{[58 / 6869}$ in terms of the the group velocity of the hole quasi-particle $v_{h}=\partial \omega_{0} / \partial k_{z}^{\prime}$. Formula (60) shows that this expression is valid only in the middle of the quasi-particle band, where $k_{z}^{\prime} \gg k_{z}^{\prime \prime}$. The expression for $F_{r}$ for arbitrary values of $k_{z}^{\prime \prime} c$ is given in the Appendix A13.

The energy dependence of the final state (45) leads to the energy dependence of the GF of Eq. (53) via the functions $K\left(\mathbf{k}_{\|}, E\right), k_{z}^{\prime}\left(\mathbf{k}_{\|}, E\right)$, and $k_{z}^{\prime \prime}\left(\mathbf{k}_{\|}, E\right)$. Now let us assume that the matrix element slowly varies with energy

$$
\left|\mathrm{M}\left(\mathbf{k}_{\|}, E\right)\right|^{2} \approx\left|\mathrm{M}\left(\mathbf{k}_{\|}, E_{0}\right)\right|^{2}
$$

and concentrate on the role of the decay of the final states into the solid. For the analysis of photoemission in the next section we introduce the normalized GF

$$
\begin{aligned}
\tilde{\mathcal{G}}(\hat{q}, \omega) & =\frac{\mathcal{G}(\hat{q}, \omega)}{K\left(\mathbf{k}_{\|}, E_{0}\right)} \\
& =\frac{K\left(\mathbf{k}_{\|}, E\right)}{K\left(\mathbf{k}_{\|}, E_{0}\right)} F\left(\mathbf{k}, \omega-\sigma_{\mathbf{k}_{\|}, \omega}\right)
\end{aligned}
$$

and its SF

$$
\begin{aligned}
A(\omega) & =-\frac{1}{\pi} \operatorname{Im} \tilde{\mathcal{G}}(\hat{q}, \omega)=A_{1}(\omega)-A_{2}(\omega), \\
A_{1}(\omega) & =\left(1-e^{-2 k_{0}^{\prime \prime} c}\right)\left(-\frac{1}{\pi} \operatorname{Im} I_{1}\right) \\
A_{2}(\omega) & =\left(1-e^{-2 k_{0}^{\prime \prime} c}\right)\left(-\frac{1}{\pi} \operatorname{Im} I_{2}\right) .
\end{aligned}
$$

Here $A_{1}$ and $A_{2}$ give the contributions to the photocurrent from the bulk and the surface terms of the GF of Eq. (44), respectively. This normalization facilitates the comparison with the bulk SF $A_{\mathrm{b}}(\mathbf{k}, \omega)$, which is normalized to unity.

\section{DISCUSSION}

In this section, we study the behavior of the GF 62 . and the relevant SFs 64-66), which define the shape of the photocurrent energy distribution curve (EDC) through the expression (7). We neglect the $\omega$-dependence of the effective hopping in the normal direction, and in Eq. (38) we set $T_{\mathbf{k}_{\|}, \omega} \equiv T$. We assume that $T$ is real and positive, and take it as the unit of energy. We chose the value of the parallel momentum $\mathbf{k}_{\|}$that gives $\varepsilon_{\mathbf{k}_{\|}}=-2.2 T$, in order to have the quasiparticle peaks close to the lower Hubbard band for our model of the initial state self-energy, see see next subsection. This choice highlights the quasiparticle band narrowing and the dispersion of the lower Hubbard band weight in the surface-normal direction. 


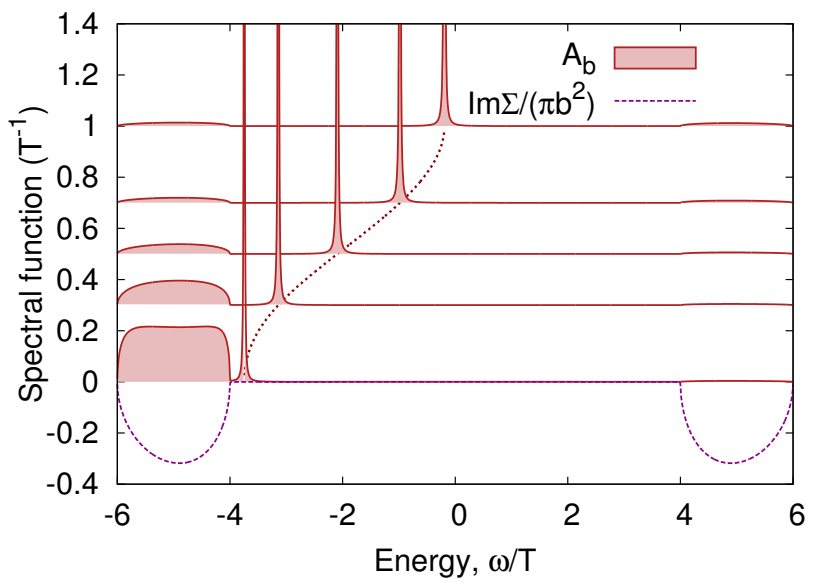

FIG. 1. (Color online) The spectral density of the bulk Green's function (38) $A_{\mathrm{b}}(\mathbf{k}, \omega+i \eta)=-\operatorname{Im} G_{b, \mathbf{k}}(\omega+i \eta) / \pi$ (31) for $\varepsilon_{\mathbf{k}_{\|}}=-2.2 T, k_{z}^{\prime} c=0,0.3 \pi, 0.5 \pi, 0.7 \pi, \pi$ (from bottom to the top), and the imaginary part of its self-energy $\operatorname{Im} \Sigma_{\mathbf{k}_{\|}, \omega+i \eta} / \pi b^{2} \sqrt[67]{6} ; b_{1}=5 T, b_{2}=b=T$. Here and below a small imaginary constant $i \eta, \eta=0.001$ is added to the energy argument in order to visualize the coherent $\delta$-function peaks in the spectral densities. Dark red dotted line shows the quasi-particle dispersion $k_{z}=\arccos \left[\left(\sigma_{\mathbf{k}_{\|}, \omega}-\omega\right) / 2 T\right]$.

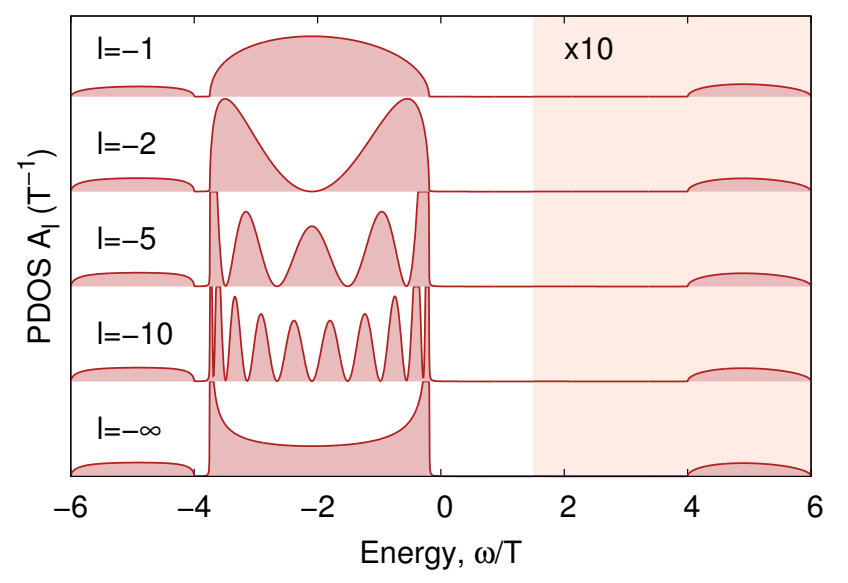

FIG. 2. (Color online) The density of states $A_{l} 70$ projected on a Bloch sum of Wannier functions located in the layer $z=l c$, Eq. 24. $\varepsilon_{\mathbf{k}_{\|}}=-2.2 T$.

\section{A. Initial states}

We adopt a simple analytic expression for the selfenergy of the bulk GF, see Eq. (37):

$$
\begin{aligned}
\Sigma_{\mathbf{k}_{\|,} \omega} & =b^{2} t_{2 b}(\omega), \\
t_{2 b}(\omega) & =1 /\left\{\omega-b_{1}^{2} /\left[\omega-b_{2}^{2} /\left(\omega-b_{1}^{2} t_{2 b}(\omega)\right)\right]\right\} \\
& =\frac{\omega^{2}-b_{1}^{2}+b_{2}^{2}+s_{t} \sqrt{\left(\omega^{2}-b_{1}^{2}-b_{2}^{2}\right)^{2}-4 b_{1}^{2} b_{2}^{2}}}{2 \omega b_{2}^{2}}
\end{aligned}
$$

where $s_{t}(\omega)=-\operatorname{sgn}\left[\operatorname{Re}\left(\omega^{2}-b_{1}^{2}-b_{2}^{2}\right)\right]$. By choosing $b_{1}>b_{2}$ and an appropriate value for $b$ we construct the function $G_{b, \mathbf{k}}(\omega)$, Eq. (38), with a three-peak structure of the SF characteristic of a Mott-Hubbard meta $\frac{37}{37}$. It has a central coherent peak at $\omega=\omega_{0}(\mathbf{k})$, see Eq. (58), and two incoherent bands over the energy intervals $\left(b_{1}-b_{2}\right)^{2}<$ $\omega^{2}<\left(b_{1}+b_{2}\right)^{2}$. Figure 1 shows the SF 31 for several values of $k_{z}$ and $b_{1}=5 T, b_{2}=b=T$. Note that the quasi-particle band is narrower than in the noninteracting case $\Sigma_{\mathbf{k}_{\|}, \omega}=0$, where its width is $4 T$. This follows from Eq. (58) because within the Hubbard gap the self-energy is approximately $\operatorname{Re}\left(\Sigma_{\mathbf{k}, \omega}\right) \sim-\alpha \omega$, with a positive coefficient $\alpha$ being weakly dependent on $\omega$ (see, e.g., Fig. 2c of Ref. 37). Then Eq. (58) gives a renormalization of the dispersion $\omega_{0}(\mathbf{k}) \sim \varepsilon_{\mathbf{k}} /(1+\alpha)$.

The incoherent bands originate from the self-energy branch cuts, where its imaginary part is a negative definite function, see the dashed line in Fig. 1. Its position does not depend on $k_{z}$ because we have chosen the coefficients $b_{1}$ and $b_{2}$ to be $k_{z}$-independent. Nevertheless, its intensity is pronouncedly momentum dependent. This dependence has the same origin as the quasiparticle band narrowing. It comes from the spectral weight redistribution, which is the consequence of the coupling between the quasi-particle and the incoherent bands. To show this, we note that the SF obeys the sum rule

$$
\int_{-\infty}^{\infty} \omega A_{\mathrm{b}}(\mathbf{k}, \omega+i 0) d \omega=\varepsilon_{\mathbf{k}}
$$

At a fixed momentum $\mathbf{k}$, the spectral density has one coherent peak situated at $\omega_{0}(\mathbf{k})$ between two incoherent bands

$$
\begin{aligned}
A_{\mathrm{b}}(\mathbf{k}, \omega+i 0) & =Z_{\mathbf{k}}\left(\omega_{0}\right) \delta\left(\omega-\omega_{0}\right)+A_{\mathrm{inc}}(\mathbf{k}, \omega), \\
A_{\mathrm{inc}}(\mathbf{k}, \omega) & =A_{\mathrm{lhb}}(\mathbf{k}, \omega)+A_{\mathrm{uhb}}(\mathbf{k}, \omega)
\end{aligned}
$$

where $A_{\mathrm{lhb}}(\mathbf{k}, \omega)$ and $A_{\mathrm{uhb}}(\mathbf{k}, \omega)$ are the low and the upper Hubbard band SFs, respectively, that yield the humps at the energies $\omega_{\mathrm{lhb} / \mathrm{uhb}} \sim \pm b_{1}$. Then, for the incoherent bands we obtain

$$
\begin{aligned}
\int_{-\infty}^{\infty} \omega A_{\mathrm{inc}}(\omega) d \omega & \sim \omega_{\mathrm{lhb}} W_{\mathrm{lhb}}(\mathbf{k})+\omega_{\mathrm{uhb}} W_{\mathrm{uhb}}(\mathbf{k}) \\
& \sim \omega_{0}(\mathbf{k})\left(1+\alpha-Z_{\mathbf{k}}\right)
\end{aligned}
$$

The spectral weights $W_{\mathrm{lhb}}(\mathbf{k})$ and $W_{\mathrm{uhb}}(\mathbf{k})$, obviously depend on k. In Eq. 68), we approximated the integrals $\int_{-\infty}^{\infty} \omega A_{\mathrm{i}}(\omega) d \omega$ by $\omega_{\mathrm{i}} W_{\mathrm{i}}(\mathbf{k})$, where " $\mathrm{i}$ " is "lhb" or "uhb". The momentum dependence of the incoherent band weight was recently observed in ARPES experiments and in the DMFT calculations for vanadates $\mathrm{Sr}_{x} \mathrm{Ca}_{1-x} \mathrm{VO}_{3} \frac{819}{\text {. }}$

It is instructive to calculate the DOS projected on the 2D Bloch sum of Wannier functions, Eq. 24, in the layer 
$z=l c:$

$$
\begin{aligned}
A_{l} & =-\frac{1}{\pi} \operatorname{Im} G_{\mathbf{k}_{\|}, l, l}(\omega) \\
G_{\mathbf{k}_{\|}, l, l} & \equiv\left\langle\left\langle a_{\mathbf{k}_{\|}, l} \mid a_{\mathbf{k}_{\|}, l}^{\dagger}\right\rangle\right\rangle_{\omega} \\
& =\frac{1}{N_{\perp}} \sum_{p_{1}, p_{2}} e^{i\left(p_{1}-p_{2}\right) l c} G_{\mathbf{k}_{\|}, p_{1}, p_{2}}(\omega),
\end{aligned}
$$

where $G_{\mathbf{k}_{\|}, p_{1}, p_{2}}(\omega)$ is the GF of the semi-infinite system given by Eq. (44). The integrals over $p_{1}$ and $p_{2}$ are calculated using Eq. (51), and the result looks very simple:

$$
G_{\mathbf{k}_{\|}, l, l}=g_{\mathbf{k}_{\|}}(\omega)\left\{1-\left[T g_{s}\left(\omega-\sigma_{\mathbf{k}_{\|}, \omega}, T\right)\right]^{2|l|}\right\},
$$

where $g_{s}(\omega)$ is given by Eq. (57). The function $g_{\mathbf{k}_{\|}}(\omega)$, Eqs. (42) and (A9), is the bulk value of the layer function $G_{\mathbf{k}_{\|}, l, l}, l \rightarrow \infty$. Figure 2 shows the result for layers at different depths. We see that the projected DOS has rather peculiar dependence on $l$ (cf. Sec. 4 and Fig. 1 of Ref. 67), and its convergence to the bulk shape is slow. Strong oscillations of the DOS near the surface were also documented in ab initio calculations, see, e.g., Fig. 4(d) in Ref. 70.

\section{B. Final states}

In order to take into account the inelastic scattering of electrons in the LEED experiment Slater ${ }^{[53}$ proposed to add an imaginary term to the potential energy. The Schrödinger equation then reads

$$
\left[-\frac{1}{2} \nabla^{2}+V(\mathbf{x})-i V_{\mathrm{i}}\right] \varphi_{>}(\mathbf{x})=E \varphi_{>}(\mathbf{x})
$$

where $V(\mathbf{x})$ is the periodic potential inside the solid. The optical potential $V_{\mathrm{i}}$ may be considered an approximation for the imaginary part of the electron self-energy.

Following Ref. 171, we start from the solution of the unperturbed problem

$$
\left[-\frac{1}{2} \nabla^{2}+V(\mathbf{x})\right] \varphi_{0}(\mathbf{k}, \mathbf{x})=\epsilon(\mathbf{k}) \varphi_{0}(\mathbf{k}, \mathbf{x})
$$

in Wannier representation

$$
\varphi_{0}(\mathbf{k}, \mathbf{x})=\frac{1}{\sqrt{N_{\|} N_{\perp}}} \sum_{\mathbf{R}} \mathrm{e}^{i \mathbf{k R}} w_{f}(\mathbf{x}-\mathbf{R})
$$

and search the solution of the perturbed problem 771 in the form

$$
\varphi_{>}(\mathbf{x})=\sum_{\mathbf{R}} \Psi(\mathbf{R}) w_{f}(\mathbf{x}-\mathbf{R}) .
$$

Then the modulating function $\Psi(\mathbf{R})$ is the solution of the equation

$$
\left[\hat{\epsilon}(-i \nabla)-i V_{\mathrm{i}}\right] \Psi(\mathbf{x})=E \Psi(\mathbf{x}) .
$$
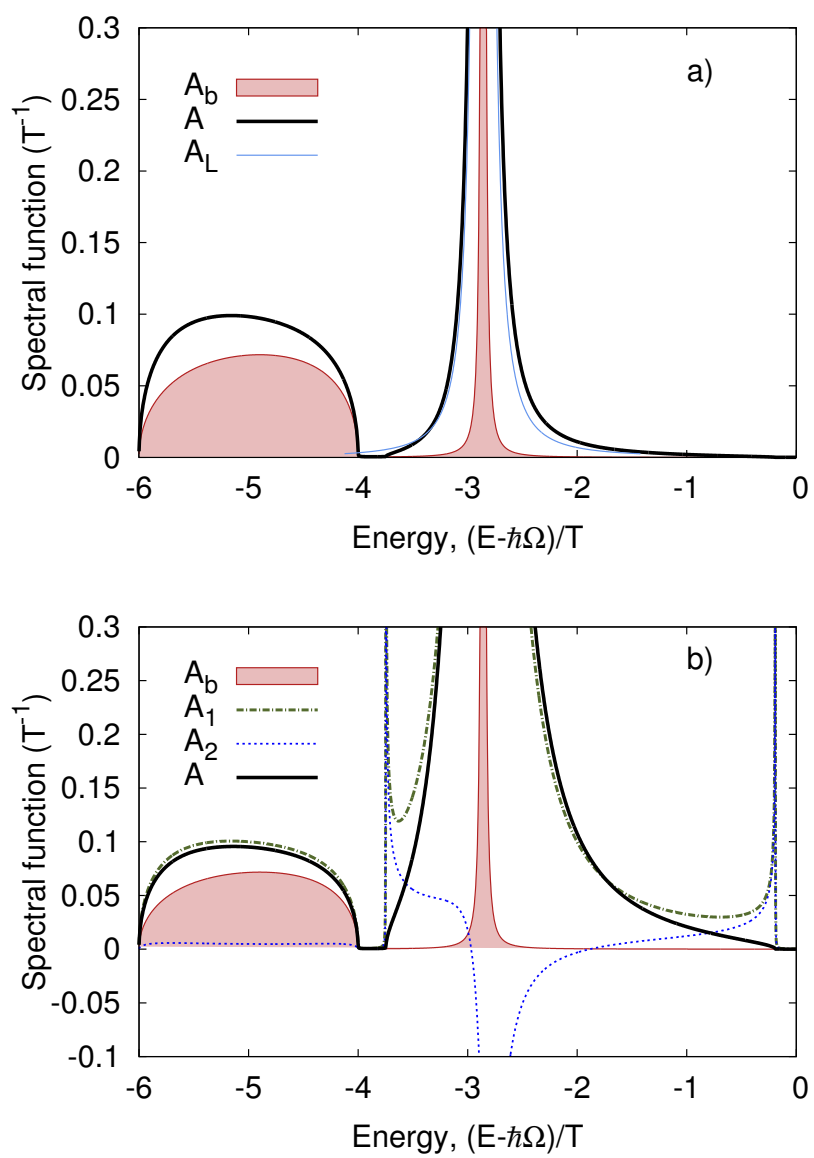

FIG. 3. (Color online) The spectral density $A(\omega+i \eta)$ 64 compared with the spectral density $A_{\mathrm{b}}(\mathbf{k}, \omega+i \eta)(31)$ of the bulk Green's function (38) shown in Fig. 1 for (a) $V_{\mathrm{i}}=0.1 T$ and (b) $V_{\mathrm{i}}=T$. The parameters are $\epsilon\left(\mathbf{k}_{0}\right)=\hbar \Omega-2.86 T$, $\varepsilon_{\mathbf{k}_{\|}}=-2.2 T \epsilon_{z}^{\prime}=10 T / c, k_{0} c=0.36 \pi$. The contributions coming from the bulk $\left[A_{1}(\omega+i \eta)\right.$, Eq. [65)], and the surface $\left[A_{2}(\omega+i \eta)\right.$, Eq. 66 $]$ terms of the Green's function 44 are also shown. Thin cornflower blue line in the upper panel shows the spectral density behavior near the ( $\delta$-functional) coherent peak of the bulk Green's function Lorentzian 59

Here $\hat{\epsilon}(-i \nabla)$ is a differential operator obtained from the function $\epsilon(\mathbf{k})$ by the substitution $\mathbf{k} \rightarrow-i \nabla$. Thus, Eq. (72) is a Schrödinger equation for $\Psi(\mathbf{x})$, in which the perturbation $-i V_{\mathrm{i}}$ is the potential energy, while the kinetic energy operator is derived from the band structure $\epsilon(\mathbf{k})$ of the unperturbed problem.

Substituting $\Psi(\mathbf{x})=\exp (i \mathbf{k x})$ with complex $\mathbf{k}=\mathbf{k}_{\|}-$ $\left(k_{z}^{\prime}+i k_{z}^{\prime \prime}\right) \mathbf{n}$ (in LEED, $\mathbf{k}_{\perp}$ points into the crystal), we obtain

$$
\epsilon\left[\mathbf{k}_{\|}-\left(k_{z}^{\prime}+i k_{z}^{\prime \prime}\right) \mathbf{n}\right]=E+i V_{\mathrm{i}},
$$

which allows to find the components of complex $\mathbf{k}$-vector from the analytical continuation of the function $\epsilon(\mathbf{k})$ into the complex energy plane. 


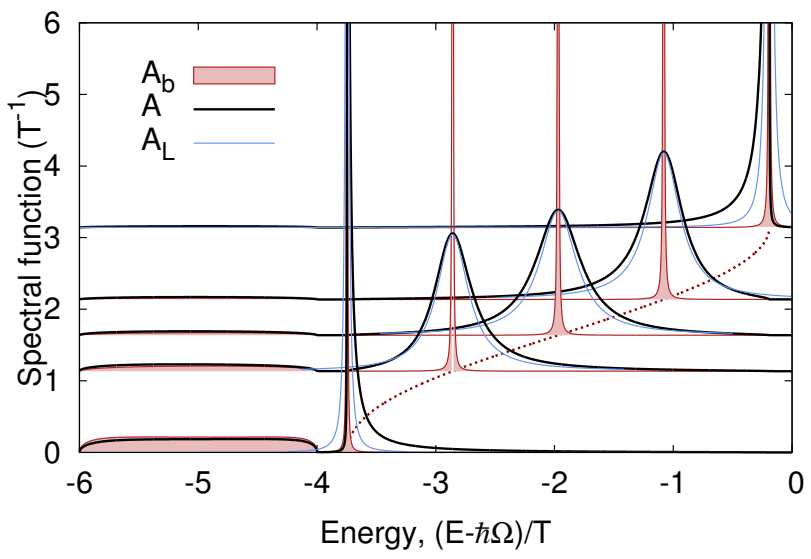

FIG. 4. (Color online) The spectral density $A(\omega+i \eta) 64$ for different photon energies $\hbar \Omega$. The lowest curve corresponds to $k_{0} c=0$, and the topmost one to $k_{0} c=\pi$. The energy step between the curves is $\hbar \Delta \Omega=0.89 T$ and $V_{\mathrm{i}}=T$. The notation is the same as in the previous figures.

\section{Final state energy far from the gap}

When the energies of photoelectrons $E$ are far from the gaps in the unoccupied spectrum $\epsilon(\mathbf{k})$, we may write

$$
\begin{aligned}
\epsilon\left[\mathbf{k}_{\|}-\left(k_{z}^{\prime}+i k_{z}^{\prime \prime}\right) \mathbf{n}\right] & \approx \epsilon\left(\mathbf{k}_{0}\right)+\epsilon_{z}^{\prime}\left(\delta+i k_{z}^{\prime \prime}\right) \\
\mathbf{k}_{0} & \equiv \mathbf{k}_{\|}-k_{0} \mathbf{n}, \\
\epsilon_{z}^{\prime} & \equiv-\left.\frac{\partial \epsilon(\mathbf{k})}{\partial k_{z}}\right|_{\mathbf{k}=\mathbf{k}_{0}}>0, \\
\delta & \equiv k_{z}^{\prime}-k_{0} .
\end{aligned}
$$

Equation (73) then gives $k_{z}$ with a constant imaginary part and a real part that linearly depends on energy

$$
\begin{aligned}
& k_{z}^{\prime}=k_{0}+\frac{E-\epsilon\left(\mathbf{k}_{0}\right)}{\epsilon_{z}^{\prime}\left(\mathbf{k}_{0}\right)}, \\
& k_{z}^{\prime \prime}=\frac{V_{i}}{\epsilon_{z}^{\prime}\left(\mathbf{k}_{0}\right)} .
\end{aligned}
$$

Figures 3 and 4 show typical EDCs in this regime in comparison with the bulk SF. The coherent peaks, which are $\delta$-functions in our approximation for the initial states, transform into Lorentzians, whose width according to Eq. (61) is proportional to $k_{z}^{\prime \prime}$ and to the quasiparticle group velocity perpendicular to the surface 68169 . The broadening is invisible for the incoherent part, but the dependence of the real part of the wave vector $k_{z}^{\prime}$ on energy (74) leads to deviations of the EDC shape from the $\mathrm{SF}$ as a result of the dispersion of the intensity of the incoherent part with $k_{z}^{\prime}$.

\section{Final states near the Bragg gap}

Now we consider the case when the energy of photoelectrons is close to a gap in the spectrum $\epsilon(\mathbf{k})$, i.e.
$\mathbf{k}=\mathbf{k}_{\|}+\left(\frac{G}{2}+\delta\right) \mathbf{n}$ is near a Brillouin zone boundary, $G \mathbf{n}$ being a reciprocal lattice vector. Then $\epsilon(\mathbf{k})$ can be approximated as

$$
\epsilon(\mathbf{k}) \approx E_{G} \pm \sqrt{W^{2}+\left(\epsilon_{z}^{\prime}\right)^{2} \delta^{2}},
$$

where $E_{G}$ and $W$ are the center and the half-width of the gap, and $\epsilon_{z}^{\prime}$ is some positive value, which plays the role of a "bare group velocity" in the absence of coupling between waves with $\mathbf{k}$ and $\mathbf{k}+G \mathbf{n}$. Equation $(73)$ now gives

$$
\begin{aligned}
k_{z}^{\prime} & =\frac{G}{2}+\delta, \\
\delta & =\operatorname{sgn}(\Delta E) \frac{\sqrt{R(\Delta E)+\left(\Delta E-V^{2}-W^{2}\right)}}{\epsilon_{z}^{\prime}}, \\
k_{z}^{\prime \prime} & =\frac{\sqrt{R(\Delta E)-\left(\Delta E-V^{2}-W^{2}\right)}}{\epsilon_{z}^{\prime}} \\
\Delta E & \equiv E-E_{G}, \\
R(\Delta E) & \equiv \sqrt{\left(\Delta E-V^{2}-W^{2}\right)^{2}+4 V^{2}(\Delta E)^{2}} .
\end{aligned}
$$

Figure 5(a) shows that both real and imaginary parts of $k_{z}$ become energy dependent. The dependences $(76)$ and (77) are smoothed by the optical potential $V_{\mathrm{i}}$. Figure 5 (b) shows that for small values of $V_{\mathrm{i}}$ the EDC from the incoherent band may substantially deviate from the SF. This may be important for the interpretation of the lowenergy ARPES, where the inelastic scattering is relatively weak.

\section{E. Small inelastic mean free path.}

It is instructive to consider the limit $k_{z}^{\prime \prime} c \rightarrow-\infty$, in which case the final state is strongly localized near the surface. Let us consider the bulk contribution $I_{1}$ to the GF, see Eq. (47). In this limit, the only non-vanishing part is $R_{2}$, see Eqs. (52) and (A11), which yields

$$
I_{1}=\frac{S_{\omega}}{\sqrt{\left(\omega-\sigma_{\mathbf{k}_{\|}, \omega}\right)^{2}-4 T^{2}}},
$$

where

$$
S_{\omega} \equiv \operatorname{sgn}\left[\operatorname{Re}\left(\omega-\sigma_{\mathbf{k}_{\|}, \omega}\right)\right] .
$$

For the coherent band, the self-energy is real. Then the SF $A_{1}$, which is proportional to the imaginary part of $I_{1}$, see Eq. 65 , is nonzero for $\left(\omega-\sigma_{\mathbf{k}_{\|}, \omega}\right)^{2}-4 T^{2}<0$. Near the points $\omega-\sigma_{\mathbf{k}_{\|}, \omega}= \pm 2 T$, the bulk contribution $A_{1}$ to the SF has horn-like singularities typical of the 1D density of states (cf. the lowest curve in Fig. 2). These "horns" are cancelled by the same singularities in the surface term, see Eq. 49):

$$
I_{2}=\frac{S_{\omega}\left[\omega-\sigma_{\mathbf{k}_{\|}, \omega}-S_{\omega} \sqrt{\left(\omega-\sigma_{\mathbf{k}_{\|}, \omega}\right)^{2}-4 T^{2}}\right]^{2}}{4 T^{2} \sqrt{\left(\omega-\sigma_{\mathbf{k}_{\|}, \omega}\right)^{2}-4 T^{2}}} .
$$



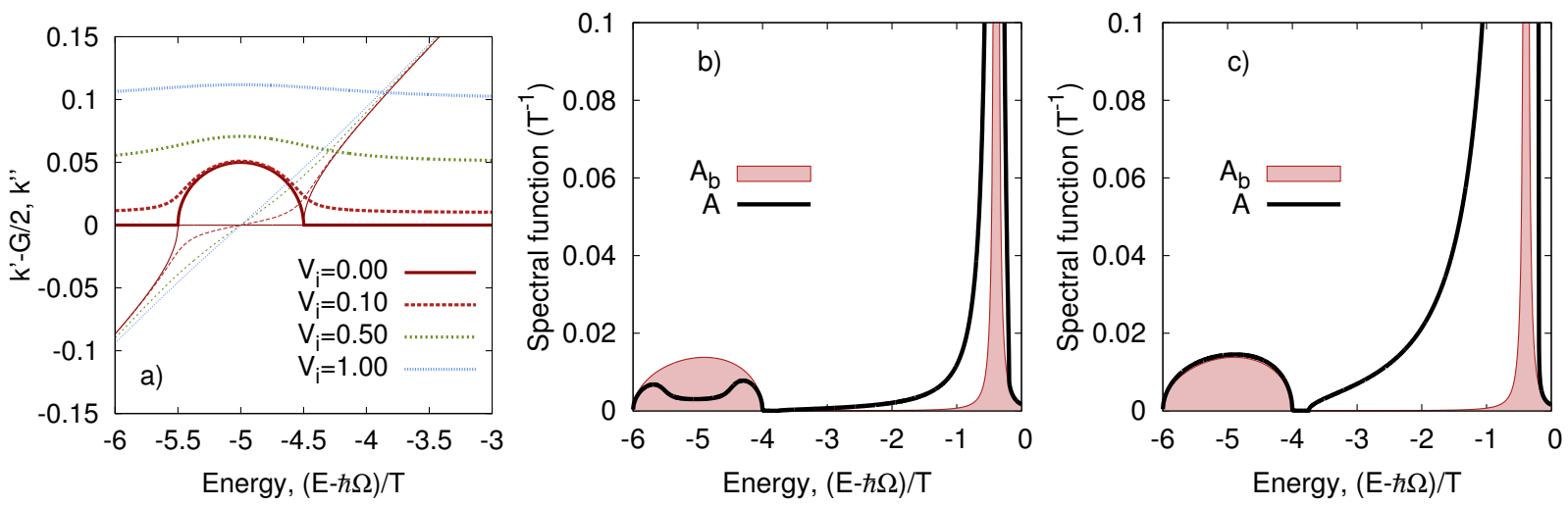

FIG. 5. (Color online) a) $k_{z}^{\prime}(76)$ - thin lines, and $k_{z}^{\prime \prime} \sqrt{77}$ - thick lines for various values of "optic potential" $V_{i} ; E_{G}=\hbar \Omega-5 T$, $\varepsilon_{\mathbf{k}_{\|}}=-2.2 T, W=0.5 T, \epsilon_{z}^{\prime}=10 T / c$. The spectral density $A(\omega+i \eta)$ compared with the spectral density of the bulk Green's function (38) shown in Fig. 1 for b) $V_{i}=0.1 T$, and c) $V_{i}=T$. The energy $E_{0}=\hbar \Omega$ is used for the normalization in Eqs. $64-66$

Substituting both expressions into (47), we obtain

$$
\begin{aligned}
& \mathcal{G}(\hat{q}, \omega) \propto\left(I_{1}-I_{2}\right) \\
& =\frac{\omega-\sigma_{\mathbf{k}_{\|}, \omega}-S_{\omega} \sqrt{\left(\omega-\sigma_{\mathbf{k}_{\|}, \omega}\right)^{2}-4 T^{2}}}{2 T^{2}} .
\end{aligned}
$$

It is clear that the imaginary part of this expression as a function of $\omega$ has the semi-elliptical form of the local DOS at the edge site of a semi-infinite chain (cf. the topmost curve in Fig. 2). Equation (79) follows from the general formula 53 in the limit $e^{-k_{z}^{\prime \prime} c} \rightarrow 0$. Figure 3(b) demonstrates that a similar cancellation happens also for finite $k_{z}^{\prime \prime}$. Thus, the account of surface terms in the initial state GF of Eq. (44) is crucial for the coherent contribution but less important for the incoherent band.

\section{CONCLUDING REMARKS}

In strongly correlated systems, the conventional understanding of the solid as a Fermi-liquid of quasiparticles breaks down in the sense that a considerable part of the spectral weight transfers from the quasiparticle peak to the incoherent band. This occurs because the removal of an electron from an $N$-electron state creates a superposition of $(N-1)$-electron eigenstates with a spread of energies. Thus, the electronic structure of strongly correlated system is described by a momentum-dependent spectral function $A_{\mathrm{b}}(\mathbf{k}, \omega)$ (31) rather than a quasiparticle energy $\varepsilon_{\mathbf{k}}$. The incoherent bands in the spectral function of the ground state come from the correlated motion of electrons expressed as the imaginary part of the self-energy. In ARPES, these bands are observed as structureless humps apart from pronounced quasiparticle peaks.

ARPES data provide the information about both initial and final states of the photoemission process. Both kinds of states characterize the solid under study, and they are solutions of the Schrödinger equation with the same Hamiltonian. Using the sudden approximation, we have shown how the spectra depend on physical properties of the initial and final states. First, we recast the well-known mean-field theory expression for the photocurrent in the one-step approach as a formula for a DOS-function projected onto a surface-localized electron state $\chi$, Eq. (10). The wave function $\chi(\mathbf{r})$ decays into the solid owing to the spatial decay of the time-reversed LEED function, and, at the same time, it rapidly vanishes in the vacuum owing to the confinement of the initial states. Then the many-body calculation of the ARPES intensity in the one-step approach reduces to the calculation of a spectral function of the two-time retarded GF for an operator that creates an electron in the state $\chi$.

Further, we make use of the Wannier representation and obtain the GF for a semi-infinite system out of the GF of an infinite system. This approach is especially advantageous for strongly correlated systems. For the simplest case of a one-band Mott-Hubbard system and neglecting the modification of the crystal potential at the surface we have obtained an analytical result. Combined with modern numerical methods, our approach is fully applicable to realistic models of surfaces.

Furthermore, for the present model we have obtained an analytical expression for the photocurrent assuming that the inelastic scattering in the final state can be described by a mean free path. Here we approximated the LEED function inside the solid by a single evanescent wave. This is not a serious limitation, which may be easily lifted within the present formalism. Expressions (7) and (53) explicitly relate the energy distribution of the photocurrent to the bulk electronic structure.

The analysis of the expressions reveal the following features of the photocurrent: (i) As in the mean-field theory $\sqrt{58|68| 69}$, the quasi-particle pole of the bulk Green's functions gives rise to a resonance, whose width is proportional to the imaginary part of the wave vector $k_{z}^{\prime \prime}$ and to the group velocity of the hole perpendicular to the sur- 
face. (ii) For the incoherent band, even if its energy range is $\mathbf{k}$-independent, as is the case in most DMFT theories, its spectral weight turns out momentum-dependent $\frac{89}{}$. Apart form the obvious $\mathbf{k}_{\|}$-dependence of the intensity, this manifests itself in the photon energy dependence of the EDC. This reflects in the first place the $\mathbf{k}$-dependence of the initial-state spectral function $A_{\mathrm{b}}(\mathbf{k}, \omega)$, Eq. 31, but it may also involve more complicated matrix element effects. This happens already in the simplest case when the final-state decay rate $k_{z}^{\prime \prime}$ is constant over the whole EDC energy range. However, when the energy passes through a gap in the final-state spectrum, where $k_{z}^{\prime \prime}$ rapidly changes with energy, EDC becomes dramatically distorted with respect to the underlying spectral function $A_{\text {inc }}(\mathbf{k}, \omega)$. Furthermore, interesting interference effects are expected when the LEED function has several evanescent components with different decay rates 72 .

In this work, we have considered rather simple models of both initial and final states. The present formalism opens a way to study ARPES for more realistic models of strongly correlated electron systems, such as LDA+DMFT ${ }^{[26] 36}$, LDA + Gutzwiller method ${ }^{73} 75$, LDA-based many-band Hubbard model76/77, embeddedcluster quantum chemistry calculations ${ }^{78779}$, etc. In addition, a more accurate treatment of final-state effects can be implemented $57 / 58 / 72$. Owing to the simplicity of the Wannier representation, the present formalism can be straightforwardly extended to two-photon and pumpprobe photoemission $80 \mid 81$.

\section{ACKNOWLEDGMENTS}

NATO (Belgium), Grant No. SfP-984735 is acknowledged with gratitude. This work was supported by the Spanish Ministry of Economy and Competitiveness MINECO (Project No. FIS2013-48286-C2-1-P).

\section{Appendix A: Details of derivation}

Let us consider the double integral in Eq. (4)

$$
\begin{aligned}
I & =\iint d^{3} \mathbf{x}_{1} d^{3} \mathbf{x}_{2}\left[\varphi_{1}^{\prime}+i \varphi_{1}^{\prime \prime}\right] \hat{O}_{1} G_{1,2}^{\prime \prime} \hat{O}_{2}\left[\varphi_{2}^{\prime}-i \varphi_{2}^{\prime \prime}\right] \\
& =\iint_{\mathbf{x}_{1,2} \subset \mathcal{S}} d^{3} \mathbf{x}_{1} d^{3} \mathbf{x}_{2}\left[\varphi_{1}^{\prime} \hat{O}_{1} G_{1,2}^{\prime \prime} \hat{O}_{2} \varphi_{2}^{\prime}+\varphi_{1}^{\prime \prime} \hat{O}_{1} G_{1,2}^{\prime \prime} \hat{O}_{2} \varphi_{2}^{\prime \prime}+i\left(\varphi_{1}^{\prime \prime} \hat{O}_{1} G_{1,2}^{\prime \prime} \hat{O}_{2} \varphi_{2}^{\prime}-\varphi_{1}^{\prime} \hat{O}_{1} G_{1,2}^{\prime \prime} \hat{O}_{2} \varphi_{2}^{\prime \prime}\right)\right]
\end{aligned}
$$

where we use the simplified notations $\varphi_{i} \equiv \varphi_{>}\left(\mathbf{x}_{i}, \hat{q}, E\right), \hat{O}_{i} \equiv \hat{O}\left(\mathbf{x}_{i}\right), G_{1,2} \equiv G\left(\mathbf{x}_{1}, \mathbf{x}_{2}, E-\hbar \Omega\right)$. The term in the last row, i.e. the imaginary part of the integral, vanishes because of the GF symmetry property (5), and the restriction of the integration ranges by the region inside the solid. The obtained expression we compare with

$$
\begin{aligned}
\operatorname{Im} I_{2} & =\operatorname{Im}\left(\iint d^{3} \mathbf{x}_{1} d^{3} \mathbf{x}_{2} \varphi_{1} \hat{O}_{1} G_{1,2} \hat{O}_{2} \varphi_{2}^{*}\right) \\
& =\iint_{\mathbf{x}_{1,2} \subset \mathcal{S}} d^{3} \mathbf{x}_{1} d^{3} \mathbf{x}_{2}\left[-\varphi_{1}^{\prime} \hat{O}_{1} G_{1,2}^{\prime} \hat{O}_{2} \varphi_{2}^{\prime \prime}+\varphi_{1}^{\prime \prime} \hat{O}_{1} G_{1,2}^{\prime \prime} \hat{O}_{2} \varphi_{2}^{\prime \prime}+\varphi_{1}^{\prime} \hat{O}_{1} G_{1,2}^{\prime \prime} \hat{O}_{2} \varphi_{2}^{\prime}+\varphi_{1}^{\prime \prime} \hat{O}_{1} G_{1,2}^{\prime} \hat{O}_{2} \varphi_{2}^{\prime}\right] \\
& =\iint_{\mathbf{x}_{1,2} \subset \mathcal{S}} d^{3} \mathbf{x}_{1} d^{3} \mathbf{x}_{2}\left(\varphi_{1}^{\prime} \hat{O}_{1} G_{1,2}^{\prime \prime} \hat{O}_{2} \varphi_{2}^{\prime}+\varphi_{1}^{\prime \prime} \hat{O}_{1} G_{1,2}^{\prime \prime} \hat{O}_{2} \varphi_{2}^{\prime \prime}\right)=I
\end{aligned}
$$

where we have again exploit the property (5), and the restriction of the integration range by the volume inside the solid due to confinement of the initial state. We thus obtain

$$
I=\operatorname{Im} \mathcal{G}(E-\hbar \Omega)
$$

where

$$
\mathcal{G}(\hat{q}, \omega) \equiv\left\langle\left\langle\int d^{3} \mathbf{x}_{1} \varphi_{>}\left(\mathbf{x}_{1}, \hat{q}, E\right) \hat{O}\left(\mathbf{x}_{1}\right) \hat{\psi}\left(\mathbf{x}_{1}\right) \mid \int d^{3} \mathbf{x}_{2} \hat{\psi}^{\dagger}\left(\mathbf{x}_{2}\right) \hat{O}\left(\mathbf{x}_{2}\right) \varphi_{>}^{*}\left(\mathbf{x}_{2}, \hat{q}, E\right)\right\rangle\right\rangle_{\omega}=\left\langle\left\langle\hat{C} \mid \hat{C}^{\dagger}\right\rangle\right\rangle_{\omega}
$$

with $\hat{C}_{\sigma}$ given by Eq. $\sqrt{9}, \omega \equiv E-\hbar \Omega$.

Now we can use the expression 14 for $\varphi_{>}\left(\mathbf{x}_{i}, \hat{q}, E\right)$ to write 


$$
\begin{aligned}
\hat{C} & =\sum_{\mathbf{R}_{\|}, l, \alpha} \iint d^{2} \mathbf{x}_{\|} \int d z e^{-i \mathbf{k}_{\|} \mathbf{x}_{\|}} U\left(\mathbf{x}_{\|}, z, \hat{q}, E\right) \hat{O}(\mathbf{x}) w_{\alpha}\left[\mathbf{x}_{\|}-\mathbf{R}_{\|}+(z-l c) \mathbf{n}-\mathbf{s}\right] a_{\mathbf{R}_{\|}, l, \alpha} \\
& =\sum_{\mathbf{R}_{\|}, l, \alpha} \iint d^{2} \mathbf{x}_{\|} \int d z e^{-i \mathbf{k}_{\|}\left(\mathbf{x}_{\|}+\mathbf{R}_{\|}\right)} U\left(\mathbf{x}_{\|}, z, \hat{q}, E\right) \hat{O}(\mathbf{x}) w_{\alpha}\left[\mathbf{x}_{\|}+(z-l c) \mathbf{n}-\mathbf{s}\right] a_{\mathbf{R}_{\|}, l, \alpha} \\
& =\sum_{l, \alpha} \int d z \iint d^{2} \mathbf{x}_{\|} \varphi_{>}(\mathbf{x}, \hat{q}, E) \hat{O}(\mathbf{x}) w_{\alpha}\left[\mathbf{x}_{\|}+(z-l c) \mathbf{n}-\mathbf{s}\right] \sum_{\mathbf{R}_{\|}} e^{-i \mathbf{k}_{\|} \mathbf{R}_{\|}} a_{\mathbf{R}_{\|}, l, \alpha},
\end{aligned}
$$

and obtain Eq. (17).

Substituting the Eq. 15 into (17) we rewrite it in the form

$$
\hat{C}=\sqrt{N_{\|}} \sum_{m, l, \alpha} \int d z \iint d^{2} \mathbf{x}_{\|} e^{-i\left[\mathbf{k}_{\|} \mathbf{x}_{\|}+k_{\perp, m}^{*}\left(z-z_{0}\right)\right]} u_{m}\left(\mathbf{x}, \mathbf{k}_{\|}, E\right) \hat{O}(\mathbf{x}) w_{\alpha}\left[\mathbf{x}_{\|}+(z-l c) \mathbf{n}-\mathbf{s}\right] a_{\mathbf{k}_{\|}, l, \alpha}
$$

that gives Eqs. 18, 19

For the one-band model considered in Sec. III, and the final sate given by Eq. 45 we have

$$
\hat{C}=\sqrt{\frac{N_{\|}}{N_{\perp}}} \mathrm{M}\left(\mathbf{k}_{\|}, E\right) \sum_{p} e^{i p z_{0}} \Delta_{p} a_{\mathbf{k}_{\|}, p, \alpha} .
$$

For the GF of Eq. Eq. 26) we have

$$
\mathcal{G}(\hat{q}, \omega)=\left|\mathrm{M}\left(\mathbf{k}_{\|}, E\right)\right|^{2} N_{\|}\left\{\frac{1}{N_{\perp}} \sum_{p}\left|\Delta_{p}\right|^{2} G_{b, \mathbf{k}}(\omega)-\frac{1}{N_{\perp}^{2} g_{\mathbf{k}_{\|}}(\omega)} \sum_{p_{1}, p_{2}} e^{i\left(p_{1}-p_{2}\right) z_{0}} \Delta_{p_{1}} \Delta_{p_{2}}^{*} G_{b, \mathbf{k}_{1}}(\omega) G_{b, \mathbf{k}_{2}}(\omega)\right\},
$$

where $\mathbf{k}_{i}=\mathbf{k}_{\|}+p_{i} \mathbf{n}, G_{b, \mathbf{k}}(\omega)$ is given by Eq. 38 . Usual substitution $\left(1 / N_{\perp}\right) \sum_{p} \cdots \rightarrow(1 / 2 \pi c) \int_{-\pi / c}^{\pi / c} \cdots d p$ gives Eq. 47. The integrals $I_{1}$ (48), and $I_{21}(50$ are calculated using the substitution

$$
z=e^{i p c}, d z=i c e^{i p c} d p, d p=-i \frac{d z}{c z}
$$

then

$$
\begin{aligned}
I_{1}\left(\mathbf{k}_{\|}, \omega\right) & =-\frac{i}{2 \pi} \oint_{|z|=1} \frac{d z}{\left(1-z_{k} z\right)\left(z-z_{k}^{*}\right)} \frac{1}{\omega-\sigma_{\mathbf{k}_{\|}, \omega}+T_{\mathbf{k}_{\|}, \omega}\left(z+\frac{1}{z}\right)} \\
& =-\frac{i}{2 \pi} \oint_{|z|=1} \frac{d z}{\left(1-z_{k} z\right)\left(z-z_{k}^{*}\right)} \frac{z}{T_{\mathbf{k}_{\|}, \omega}\left(z-z_{S}\right)\left(z-z_{-S}\right)}
\end{aligned}
$$

where $z_{k} \equiv e^{-i k_{z}^{\prime}-k_{z}^{\prime \prime} c}, \sigma_{\mathbf{k}_{\|}, \omega} \equiv \varepsilon_{\mathbf{k}_{\|}}+\Sigma_{\mathbf{k}_{\|}, \omega}$

$$
z_{ \pm S}=-\frac{\omega-\sigma_{\mathbf{k}_{\|}, \omega} \mp S_{\omega} \sqrt{\left(\omega-\sigma_{\mathbf{k}_{\|}, \omega}\right)^{2}-4 T_{\mathbf{k}_{\|}, \omega}^{2}}}{2 T_{\mathbf{k}_{\|}, \omega}}
$$

function $S_{\omega}$ is given by Eq. (78). Noting that $\left|z_{k}\right|,\left|z_{S}\right|<1$, we find two poles lying inside the circle $|z|<1: z_{1}=z_{k}^{*}$, and $z_{2}=z_{S}$. This gives Eq. (52), with

$$
\begin{aligned}
R_{1} & =\frac{1}{1-\left|z_{k}\right|^{2}} \frac{z_{k}^{*}}{T_{\mathbf{k}_{\|}, \omega}\left(z_{k}^{*}-z_{-S}\right)\left(z_{k}^{*}-z_{S}\right)} \\
& =\frac{1}{1-\left|z_{k}\right|^{2}} \frac{1}{\omega-\sigma_{\mathbf{k}_{\|}, \omega}+T_{\mathbf{k}_{\|}, \omega}\left(z_{k}^{*}+\frac{1}{z_{k}^{*}}\right)}, \\
R_{2} & =\frac{1}{\left(1-z_{k} z_{S}\right)\left(z_{S}-z_{k}^{*}\right)} \frac{z_{S}}{T_{\mathbf{k}_{\|}, \omega}\left(z_{S}-z_{-S}\right)} .
\end{aligned}
$$


Similarly, we have

$$
\begin{aligned}
I_{21}\left(k_{z}^{\prime}\right) & =-\frac{i}{2 \pi} \oint_{|z|=1} \frac{d z}{\left(1-z_{k} z\right)} \frac{z}{T_{\mathbf{k}_{\|}, \omega}\left(z-z_{S}\right)\left(z-z_{-S}\right)} \\
& =\frac{1}{\left(1-z_{k} z_{S}\right)} \frac{z_{S}}{T_{\mathbf{k}_{\|}, \omega}\left(z_{S}-z_{-S}\right)} .
\end{aligned}
$$

Then

$$
\begin{aligned}
I_{2} & =\frac{1}{g_{\mathbf{k}_{\|}}(\omega)} I_{21}\left(k_{z}^{\prime}\right) I_{21}\left(-k_{z}^{\prime}\right)=\frac{1}{\left(1-z_{k} z_{S}\right)} \frac{T_{\mathbf{k}_{\|}, \omega}\left(z_{S}-z_{-S}\right)}{\left(1-z_{k}^{*} z_{S}\right)}\left[\frac{z_{S}}{T_{\mathbf{k}_{\|}, \omega}\left(z_{S}-z_{-S}\right)}\right]^{2}, \\
I & =R_{1}+R_{2}-I_{2}=\frac{1}{T_{\mathbf{k}_{\|}, \omega}\left(1-\left|z_{k}\right|^{2}\right)\left(z_{k}^{*} z_{S}-1\right)\left(z_{-S}-z_{k}\right)}
\end{aligned}
$$

Above, we have taken into account that

$$
g_{\mathbf{k}_{\|}}(\omega)=\frac{1}{T_{\mathbf{k}_{\|}, \omega}\left(z_{S}-z_{-S}\right)}=\frac{S_{\omega}}{\sqrt{\left(\omega-\sigma_{\mathbf{k}_{\|}, \omega}\right)^{2}-4 T_{\mathbf{k}_{\|}, \omega}^{2}}} .
$$

Substituting $z_{k}$ and $z_{ \pm S}$ into the above expressions, we obtain the formulas for various contributions into 477)

$$
\begin{aligned}
R_{1}\left(\mathbf{k}_{\|}, \omega\right) & =\left\{\left(1-e^{-2 k_{z}^{\prime \prime} c}\right)\left[\omega-\sigma_{\mathbf{k}_{\|}, \omega}+2 T_{\mathbf{k}_{\|}, \omega} \cos \left(k_{z}^{\prime} c+i k_{z}^{\prime \prime} c\right)\right]\right\}^{-1}, \\
R_{2}\left(\mathbf{k}_{\|}, \omega\right) & =\frac{S_{\omega} T_{\mathbf{k}_{\|}, \omega} e^{k_{z}^{\prime \prime} c}}{\left[2 T_{\mathbf{k}_{\|}, \omega} \cosh k_{z}^{\prime \prime} c+\left(\omega-\sigma_{\mathbf{k}_{\|}, \omega}\right) \cos k_{z}^{\prime} c+i S_{\omega} \sin k_{z}^{\prime} c \sqrt{\left(\omega-\sigma_{\mathbf{k}_{\|}, \omega}\right)^{2}-4 T_{\mathbf{k}_{\|}, \omega}^{2}}\right] \sqrt{\left(\omega-\sigma_{\mathbf{k}_{\|}, \omega}\right)^{2}-4 T_{\mathbf{k}_{\|}, \omega}^{2}}}, \\
I_{2}\left(\mathbf{k}_{\|}, \omega\right) & =\frac{2 S_{\omega} T_{\mathbf{k}_{\|}, \omega}^{2}}{\left[\left(\omega-\sigma_{\mathbf{k}_{\|}, \omega}+S_{\omega} \sqrt{\left(\omega-\sigma_{\mathbf{k}_{\|}, \omega}\right)^{2}-4 T_{\mathbf{k}_{\|}, \omega}^{2}}\right)\left(\omega-\sigma_{\mathbf{k}_{\|}, \omega}+2 T_{\mathbf{k}_{\|}, \omega} e^{-k_{z}^{\prime \prime} c} \cos k_{z}^{\prime} c\right)-2 T_{\mathbf{k}_{\|}, \omega}^{2}\left(1-e^{-2 k_{z}^{\prime \prime} c}\right)\right]} \\
& \times \frac{1}{\sqrt{\left(\omega-\sigma_{\mathbf{k}_{\|}, \omega}\right)^{2}-4 T_{\mathbf{k}_{\|}, \omega}^{2}}} .
\end{aligned}
$$

On the other hand, we note that

$$
z_{S}=-T_{\mathbf{k}_{\|}, \omega} g_{s}\left(\omega-\sigma_{\mathbf{k}_{\|}, \omega}, T_{\mathbf{k}_{\|}, \omega}\right), \quad z_{-S}=-\frac{\omega-\sigma_{\mathbf{k}_{\|}, \omega}}{T_{\mathbf{k}_{\|}, \omega}}-z_{S}
$$

then the denominator of the second fraction of the right-hand side of $(A 8)$ is

$$
\begin{aligned}
& z_{k}^{*} z_{S} z_{-S}-\left|z_{k}\right|^{2} z_{S}-z_{-S}+z_{k}= \\
& =\frac{\omega-\sigma_{\mathbf{k}_{\|}, \omega}+2 T_{\mathbf{k}_{\|}, \omega} e^{-k_{z}^{\prime \prime} c} \cos k_{z}^{\prime} c-T_{\mathbf{k}_{\|}, \omega}^{2}\left(1-e^{-2 k_{z}^{\prime \prime} c}\right) g_{s}\left(\omega-\sigma_{\mathbf{k}_{\|}, \omega}, T_{\mathbf{k}_{\|}, \omega}\right)}{T_{\mathbf{k}_{\|}, \omega}},
\end{aligned}
$$

and $\mathcal{G}$ is given by Eq. 53 .

The behavior near the resonance frequency $\omega_{r}$ is described by the expression

$$
\begin{aligned}
F_{r}\left(\mathbf{k}_{\|}, \omega\right) & =\frac{Z\left(\omega_{r}\right) e^{k_{z}^{\prime \prime} c}}{\left(\omega-\omega_{r}\right) \cosh k_{z}^{\prime \prime} c+i \Gamma} \\
\Gamma & =2 Z\left(\omega_{r}\right)\left|T_{\mathbf{k}_{\|}, \omega_{r}}\right| \tanh k_{z}^{\prime \prime} c \sqrt{\sinh ^{2} k_{z}^{\prime \prime} c+\sin ^{2} k_{z}^{\prime} c}
\end{aligned}
$$

1 S. Hüfner, Photoelectron Spectroscopy: Principles and Applications, Advanced Texts in Physics (Springer, 2003).
2 Andrea Damascelli, Zahid Hussain, and Zhi-Xun Shen, 
"Angle-resolved photoemission studies of the cuprate superconductors," Rev. Mod. Phys. 75, 473-541 (2003).

3 W. Schattke and M.A. Van Hove, Solid-State Photoemission and Related Methods: Theory and Experiment (Wiley, 2008).

4 A. A. Kordyuk, "Arpes experiment in fermiology of quasi2d metals (review article)," Low Temperature Physics 40, 286-296 (2014).

${ }^{5}$ P. Fulde, Electron Correlations in Molecules and Solids, Springer series in solid-state sciences (Springer-Verlag, Berlin, Heidelberg, 1991).

6 Masatoshi Imada, Atsushi Fujimori, and Yoshinori Tokura, "Metal-insulator transitions," Rev. Mod. Phys. 70, 1039-1263 (1998)

G. A. Sawatzky, "Testing fermi-liquid models," Nature 342, 480-481 (1989).

$\checkmark$ M. Takizawa, M. Minohara, H. Kumigashira, D. Toyota, M. Oshima, H. Wadati, T. Yoshida, A. Fujimori, M. Lippmaa, M. Kawasaki, H. Koinuma, G. Sordi, and M. Rozenberg, "Coherent and incoherent $d$ band dispersions in $\mathrm{srvo}_{3}$, " Phys. Rev. B 80, 235104 (2009).

9 T. Yoshida, M. Hashimoto, T. Takizawa, A. Fujimori, M. Kubota, K. Ono, and H. Eisaki, "Mass renormalization in the bandwidth-controlled mott-hubbard systems $\mathrm{srvo}_{3}$ and cavo $_{3}$ studied by angle-resolved photoemission spectroscopy," Phys. Rev. B 82, 085119 (2010)

10 S. Aizaki, T. Yoshida, K. Yoshimatsu, M. Takizawa, M. Minohara, S. Ideta, A. Fujimori, K. Gupta, P. Mahadevan, K. Horiba, H. Kumigashira, and M. Oshima, "Self-energy on the low- to high-energy electronic structure of correlated metal srvo 3 ," Phys. Rev. Lett. 109, 056401 (2012)

it J. Laverock, J. Kuyyalil, B. Chen, R. P. Singh, B. Karlin, J. C. Woicik, G. Balakrishnan, and K. E. Smith, "Enhanced electron correlations at the $\mathrm{Sr}_{x} \mathrm{Ca}_{1-x} \mathrm{vO}_{3}$ surface," Phys. Rev. B 91, 165123 (2015).

12 G. Borstel, "Theoretical aspects of photoemission," Applied Physics A 38, 193-204 (1985).

13 I. Adawi, "Theory of the surface photoelectric effect for one and two photons," Phys. Rev. 134, A788-A798 (1964)

14 G. D. Mahan, "Theory of photoemission in simple metals," Phys. Rev. B 2, 4334-4350 (1970).

15 C. Caroli, D. Lederer-Rozenblatt, B. Roulet, and D. SaintJames, "Inelastic effects in photoemission: Microscopic formulation and qualitative discussion," Phys. Rev. B 8, 4552-4569 (1973)

10 Peter J. Feibelman and D. E. Eastman, "Photoemission spectroscopy ${ }^{-}$correspondence between quantum theory and experimental phenomenology," Phys. Rev. B 10, 4932-4947 (1974).

it J.B. Pendry, "Theory of photoemission," Surface Science

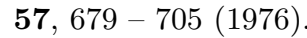

${ }^{18}$ D. W. Jepsen, F. J. Himpsel, and D. E. Eastman, "Singlestep-model analysis of angle-resolved photoemission from ni(110) and cu(100)," Phys. Rev. B 26, 4039-4051 (1982)

19 J Braun, "The theory of angle-resolved ultraviolet photoemission and its applications to ordered materials," Reports on Progress in Physics 59, 1267 (1996).

${ }^{20}$ E. E. Krasovskii, "Augmented-plane-wave approach to scattering of bloch electrons by an interface," Phys. Rev. B 70, 245322 (2004)

${ }^{21}$ M. Potthoff, J. Lachnitt, W. Nolting, and J. Braun, "Onestep model of photoemission for nonlocal potentials," physica status solidi (b) 203, 441-457 (1997)
22 C. Meyer, M. Potthoff, W. Nolting, G. Borstel, and J. Braun, "Relativistic photoemission theory for general nonlocal potentials," physica status solidi (b) 216, 10231037 (1999).

23 J. Minár, L. Chioncel, A. Perlov, H. Ebert, M. I. Katsnelson, and A. I. Lichtenstein, "Multiple-scattering formalism for correlated systems: A kkr-dmft approach," Phys. Rev. B 72, 045125 (2005)

24 J. Minár, "Correlation effects in transition metals and their alloys studied using the fully self-consistent kkr-based lsda + dmft scheme," Journal of Physics: Condensed Matter 23, 253201 (2011)

25 Antoine Georges, Gabriel Kotliar, Werner Krauth, and Marcelo J. Rozenberg, "Dynamical mean-field theory of strongly correlated fermion systems and the limit of infinite dimensions," Rev. Mod. Phys. 68, 13-125 (1996).

26 G. Kotliar, S. Y. Savrasov, K. Haule, V. S. Oudovenko, O. Parcollet, and C. A. Marianetti, "Electronic structure calculations with dynamical mean-field theory," Rev. Mod. Phys. 78, 865-951 (2006)

27 D. Vollhardt, "Dynamical mean-field theory for correlated electrons," Annalen der Physik 524, 1-19 (2012)

28 J. Braun, J. Minár, H. Ebert, M. I. Katsnelson, and A. I. Lichtenstein, "Spectral function of ferromagnetic $3 d$ metals: A self-consistent LSDA + DMFT approach combined with the one-step model of photoemission," Phys. Rev. Lett. 97, 227601 (2006).

29 J. Sánchez-Barriga, J. Fink, V. Boni, I. Di Marco, J. Braun, J. Minár, A. Varykhalov, O. Rader, V. Bellini, F. Manghi, H. Ebert, M. I. Katsnelson, A. I. Lichtenstein, O. Eriksson, W. Eberhardt, and H. A. Dürr, "Strength of correlation effects in the electronic structure of iron," Phys. Rev. Lett. 103, 267203 (2009).

30 J. Sánchez-Barriga, J. Braun, J. Minár, I. Di Marco, A. Varykhalov, O. Rader, V. Boni, V. Bellini, F. Manghi, H. Ebert, M. I. Katsnelson, A. I. Lichtenstein, O. Eriksson, W. Eberhardt, H. A. Dürr, and J. Fink, "Effects of spin-dependent quasiparticle renormalization in fe, co, and ni photoemission spectra:an experimental and theoretical study," Phys. Rev. B 85, 205109 (2012).

31 Gregory H. Wannier, "The structure of electronic excitation levels in insulating crystals," Phys. Rev. 52, 191-197 (1937)

32 Nicola Marzari and David Vanderbilt, "Maximally localized generalized wannier functions for composite energy bands," Phys. Rev. B 56, 12847-12865 (1997)

33 Nicola Marzari, Arash A. Mostofi, Jonathan R. Yates, Ivo Souza, and David Vanderbilt, "Maximally localized wannier functions: Theory and applications," Rev. Mod. Phys. 84, 1419-1475 (2012)

${ }^{34}$ P. W. Anderson, "New approach to the theory of superexchange interactions," Phys. Rev. 115, 2-13 (1959).

35 J. Hubbard, "Electron correlations in narrow energy bands," Proceedings of the Royal Society of London A: Mathematical, Physical and Engineering Sciences 276, 238-257 (1963)

${ }^{30}$ I. A. Nekrasov, K. Held, G. Keller, D. E. Kondakov, Th. Pruschke, M. Kollar, O. K. Andersen, V. I. Anisimov, and D. Vollhardt, "Momentum-resolved spectral functions of $\mathrm{SrVO}_{3}$ calculated by LDA + DMFT," Phys. Rev. B 73, $155112(2006)$.

" K. Byczuk, M. Kollar, K. Held, Y.-F. Yang, I. A. Nekrasov, Th. Pruschke, and D. Vollhardt, "Kinks in the dispersion of strongly correlated electrons," Nat. Phys. 3, 168 - 171 
(2007)

38 E. Z. Kuchinskii, I. A. Nekrasov, and M. V. Sadovskii, "Generalized dynamical mean-field theory in the physics of strongly correlated systems," Physics-Uspekhi 55, 325355 (2012).

${ }^{39}$ M Potthoff and W Nolting, "The large- $u$ hubbard model for a semi-infinite crystal: a moment approach and an energy-dependent recursion method," Journal of Physics: Condensed Matter 8, 4937 (1996)

${ }^{40}$ M. Potthoff and W. Nolting, "Metallic surface of a mott insulator $\longleftarrow$ mott insulating surface of a metal," Phys. Rev. B 60, 7834-7849 (1999)

${ }^{41}$ A. Liebsch, "Surface versus bulk coulomb correlations in photoemission spectra of $\mathrm{Srvo}_{3}$ and $\mathrm{cavo}_{3}$," Phys. Rev. Lett. 90, 096401 (2003)

${ }^{42}$ H. Ishida and A. Liebsch, "Embedding approach for dynamical mean-field theory of strongly correlated heterostructures," Phys. Rev. B 79, 045130 (2009).

43 Reza Nourafkan and Frank Marsiglio, "Surface effects in doping a mott insulator," Phys. Rev. B 83, 155116 (2011).

44 S. V. Borisenko, "“one-cubed" arpes user facility at bessy ii," Synchrotron Radiation News 25, 6-11 (2012).

45 J. Zaanen, G. A. Sawatzky, and J. W. Allen, "Band gaps and electronic structure of transition-metal compounds," Phys. Rev. Lett. 55, 418-421 (1985)

46 V. J. Emery, "Theory of high- $\mathrm{t}_{\mathrm{c}}$ superconductivity in oxides," Phys. Rev. Lett. 58, 2794-2797 (1987).

47 Arata Tanaka and Takeo Jo, "Resonant 3d, 3p and 3s photoemission in transition metal oxides predicted at $2 p$ threshold," Journal of the Physical Society of Japan 63, 2788-2807 (1994)

48 A. W. Kay, F. J. Garcia de Abajo, S.-H. Yang, E. Arenholz, B. S. Mun, N. Mannella, Z. Hussain, M. A. Van Hove, and C. S. Fadley, "Multiatom resonant photoemission," Phys. Rev. B 63, 115119 (2001).

49 Fabiana Da Pieve and Peter Krüger, "First-principles calculations of angle-resolved and spin-resolved photoemission spectra of $\operatorname{cr}(110)$ surfaces at the $2 p-3 d$ cr resonance," Phys. Rev. Lett. 110, 127401 (2013)

50 F. Da Pieve, "Fingerprints of entangled spin and orbital physics in itinerant ferromagnets via angle-resolved resonant photoemission," Phys. Rev. B 93, 035106 (2016)

51 Peter J. Feibelman, "Microscopic calculation of electromagnetic fields in refraction at a jellium-vacuum interface," Phys. Rev. B 12, 1319-1336 (1975)

52 E. E. Krasovskii, V. M. Silkin, V. U. Nazarov, P. M. Echenique, and E. V. Chulkov, "Dielectric screening and band-structure effects in low-energy photoemission," Phys. Rev. B 82, 125102 (2010).

53 J. C. Slater, "Damped electron waves in crystals," Phys. Rev. 51, 840-846 (1937)

${ }^{54}$ V. N. Strocov, H. I. Starnberg, and P. O. Nilsson, "Excited-state bands of cu determined by vleed band fitting and their implications for photoemission," Phys. Rev. B 56, 1717-1725 (1997)

5o N. Barrett, E. E. Krasovskii, J.-M. Themlin, and V. N. Strocov, "Elastic scattering effects in the electron mean free path in a graphite overlayer studied by photoelectron spectroscopy and leed," Phys. Rev. B 71, 035427 (2005)

56 E. E. Krasovskii and W. Schattke, "Surface electronic structure with the linear methods of band theory," Phys. Rev. B 56, 12874-12883 (1997)

57 X. Y. Cui, E. E. Krasovskii, V. N. Strocov, A. Hofmann, J. Schäfer, R. Claessen, and L. Patthey, "Final-state ef- fects in high-resolution angle-resolved photoemission from ni(110)," Phys. Rev. B 81, 245118 (2010).

58 E. E. Krasovskii, K. Rossnagel, A. Fedorov, W. Schattke, and L. Kipp, "Determination of the hole lifetime from photoemission: Ti $3 d$ states in tite 2 ," Phys. Rev. Lett. 98, 217604 (2007)

59 David Kalkstein and Paul Soven, "A green's function theory of surface states," Surface Science 26, 85 - 99 (1971).

60 E.N. Economou, Green's Functions in Quantum Physics (Springer-Verlag, Berlin, Heidelberg, 2006).

61 R. O. Kuzian and S.-L. Drechsler, "Exact one- and two-particle excitation spectra of acute-angle helimagnets above their saturation magnetic field," Phys. Rev. B 75, 024401 (2007)

o2 Satoshi Nishimoto, Stefan-Ludwig Drechsler, Roman Kuzian, Johannes Richter, and Jeroen van den Brink, "Interplay of interchain interactions and exchange anisotropy: Stability and fragility of multipolar states in spin- $\frac{1}{2}$ quasione-dimensional frustrated helimagnets," Phys. Rev. B 92, 214415 (2015)

${ }^{03}$ R. O. Kuzian, R. Hayn, A. F. Barabanov, and L. A. Maksimov, "Spin-polaron damping in the spin-fermion model for cuprate superconductors," Phys. Rev. B 58, 6194-6207 (1998)

${ }^{4}$ R.O. Kuzian, R. Hayn, and L.B. Litinskii, "Singularities of one-particle green's function in doped antiferromagnet," Physica B: Condensed Matter 259-261, 779 - 780 (1999).

65 R. Hayn and R. O. Kuzian, "Spectral function of one hole in several one-dimensional spin arrangements," Phys. Rev. B 62, 12156-12166 (2000).

कo Roger Haydock, "The recursive solution of the schrodinger equation," (Academic Press, 1980) pp. 215 - 294.

67 J. Henk and W. Schattke, "A subroutine package for computing green's functions of relaxed surfaces by the renormalization method," Computer Physics Communications 77, $69-83(1993)$

${ }_{0}$ H. I. Starnberg, H. E. Brauer, and P. O. Nilsson, "Lifetime broadening in bulk photoemission spectroscopy," Phys. Rev. B 48, 621-623 (1993).

${ }^{\circ y}$ N. V. Smith, P. Thiry, and Y. Petroff, "Photoemission linewidths and quasiparticle lifetimes," Phys. Rev. B 47, 15476-15481 (1993).

To A. G. Rybkin, E. E. Krasovskii, D. Marchenko, E. V. Chulkov, A. Varykhalov, O. Rader, and A. M. Shikin, "Topology of spin polarization of the $5 d$ states on w(110) and al/w(110) surfaces," Phys. Rev. B 86, 035117 (2012).

71 J. C. Slater, "Electrons in perturbed periodic lattices," Phys. Rev. 76, 1592-1601 (1949)

72 E. E. Krasovskii, V. N. Strocov, N. Barrett, H. Berger, W. Schattke, and R. Claessen, "Band mapping in the one-step photoemission theory: Multi-bloch-wave structure of final states and interference effects," Phys. Rev. B 75, 045432 (2007)

${ }^{73}$ XiaoYu Deng, Xi Dai, and Zhong Fang, "Lda + gutzwiller method for correlated electron systems," EPL (Europhysics Letters) 83, 37008 (2008).

${ }^{74}$ K. M. Ho, J. Schmalian, and C. Z. Wang, "Gutzwiller density functional theory for correlated electron systems," Phys. Rev. B 77, 073101 (2008).

75 XiaoYu Deng, Lei Wang, Xi Dai, and Zhong Fang, "Local density approximation combined with gutzwiller method for correlated electron systems: Formalism and applications," Phys. Rev. B 79, 075114 (2009)

76 R. O. Kuzian, S. Nishimoto, S.-L. Drechsler, J. Málek, 
S. Johnston, Jeroen van den Brink, M. Schmitt, H. Rosner, M. Matsuda, K. Oka, H. Yamaguchi, and T. Ito, " $\mathrm{ca}_{2} \mathrm{y}_{2} \mathrm{cu}_{5} \mathbf{O}_{10}$ : The first frustrated quasi-1d ferromagnet close to criticality," Phys. Rev. Lett. 109, 117207 (2012)

77 Claude Monney, Valentina Bisogni, Ke-Jin Zhou, Roberto Kraus, Vladimir N. Strocov, Günter Behr, Ji ři Málek, Roman Kuzian, Stefan-Ludwig Drechsler, Steve Johnston, Alexandre Revcolevschi, Bernd Büchner, Henrik M. Rønnow, Jeroen van den Brink, Jochen Geck, and Thorsten Schmitt, "Determining the short-range spin correlations in the spin-chain $\mathrm{li}_{2} \mathrm{CuO}_{2}$ and $\mathrm{cugeO}_{3}$ compounds using resonant inelastic x-ray scattering," Phys. Rev. Lett. 110, 087403 (2013)

78 Vamshi M. Katukuri, Karla Roszeitis, Viktor Yushankhai, Alexander Mitrushchenkov, Hermann Stoll, Michel van Veenendaal, Peter Fulde, Jeroen van den Brink, and
Liviu Hozoi, "Electronic structure of low-dimensional 4d5 oxides: Interplay of ligand distortions, overall lattice anisotropy, and spin-orbit interactions," Inorganic Chemistry 53, 4833-4839 (2014), pMID: 24779549.

${ }^{79}$ Satoshi Nishimoto, Vamshi M. Katukuri, Viktor Yushankhai, Hermann Stoll, Ulrich K. Roszler, Liviu Hozoi, Ioannis Rousochatzakis, and Jeroen van den Brink, "Strongly frustrated triangular spin lattice emerging from triplet dimer formation in honeycomb li2iro3," Nat Commun 7, 10273 (2016).

80 W. Schattke, E. E. Krasovskii, R. Díez Muiño, and P. M. Echenique, "Direct resolution of unoccupied states in solids via two-photon photoemission," Phys. Rev. B 78, 155314 (2008)

${ }^{1}$ J. Braun, R. Rausch, M. Potthoff, J. Minár, and H. Ebert, "One-step theory of pump-probe photoemission," Phys. Rev. B 91, 035119 (2015). 